\title{
A generic parallel computational framework of lifting wavelet transform for online engineering surface filtration
}

Yuanping $\mathrm{Xu}^{\mathrm{a}}$

Chaolong Zhang ${ }^{\mathrm{a}, \mathrm{b}}$,

18668289@qq.com

Zhijie Xu

Jiliu Zhou

Kaiwei Wang ${ }^{\mathrm{d}}$

Jian Huang

aSchool of Software Engineering, Chengdu University of Information Technology, No.24 Block 1, Xuefu Road, Chengdu 610225, People's Republic of China

${ }^{\mathbf{b}}$ School of Computing \& Engineering, University of Huddersfield, Queensgate HD1 3DH, Huddersfield, UK

'School of Computer Science, Chengdu University of Information Technology, No.24 Block 1, Xuefu Road,Chengdu 610225, People's Republic of China

dSchool of Optical Science and Engineering, Zhejiang University, Hangzhou 310027, People's Republic of China

${ }^{*}$ Corresponding author at: School of Software Engineering, Chengdu University of Information Technology, No.24 Block 1, Xuefu Road, Chengdu 610225, People's Republic of China.

Abstract

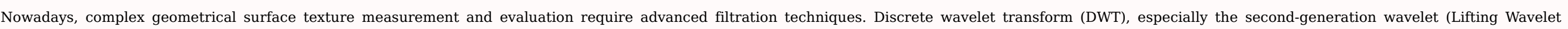

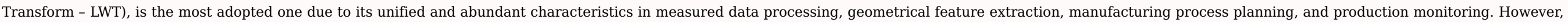

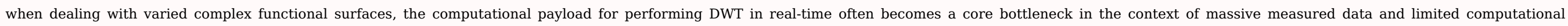

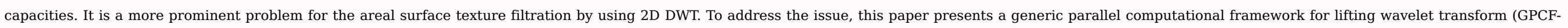

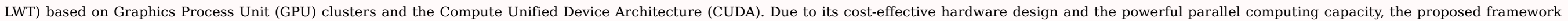

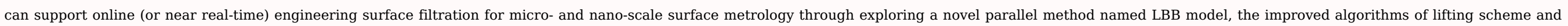

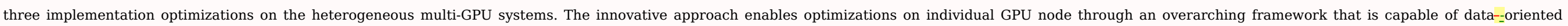

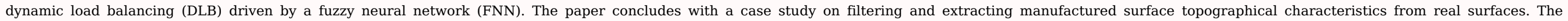
experimental results have demonstrated substantial improvements on the GPCF-LWT implementation in terms of computational efficiency, operational robustness, and task generalization

Keywords: Lifting wavelet; LBB; Surface texture Measurement; Multi-GPU; CUDA; FNN

\section{Introduction}

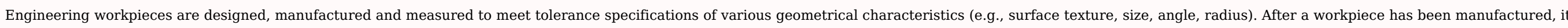

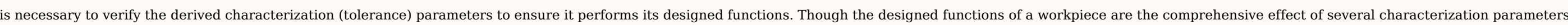

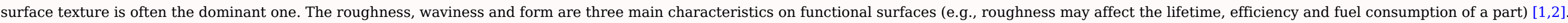

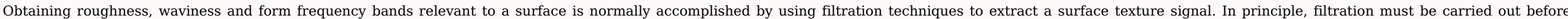




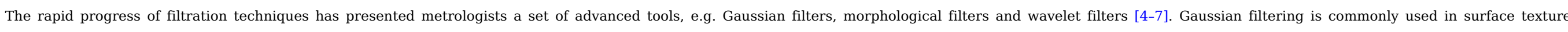

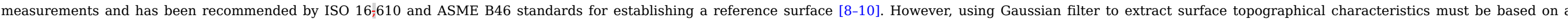

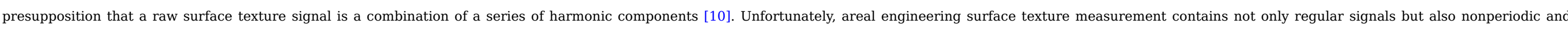

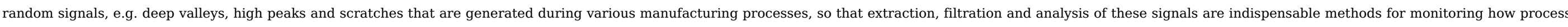

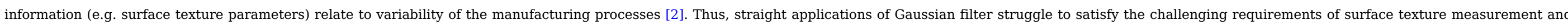

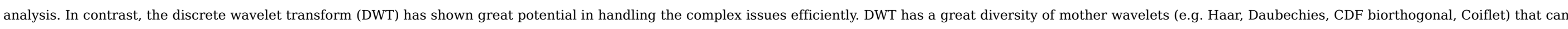
be applied according to different application purposes. Moreover, DWT has advantages on multi-resolution analysis and lower computational complexity.

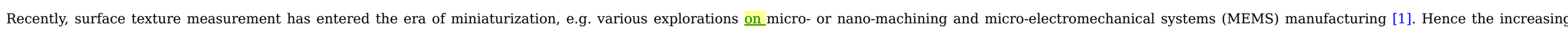

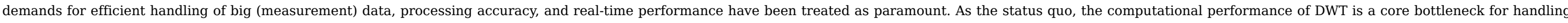
high-throughput in online (or near real-time) manner [11], especially for two-dimensional DWT (2D DWT) in areal surface texture measurement [12].

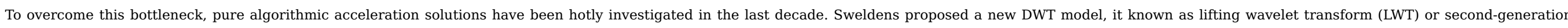

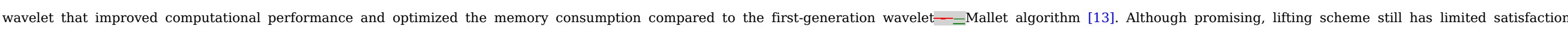

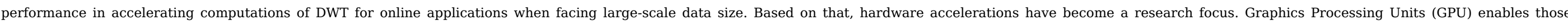

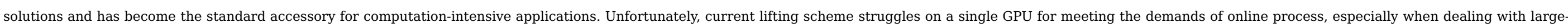
scale and dynamic datasets to define high precision requirements for surface texture metrology.

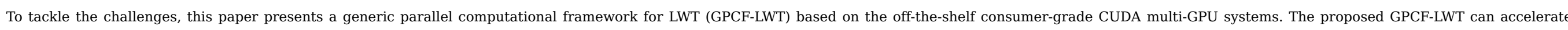

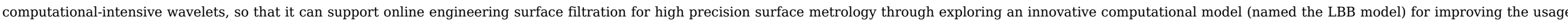

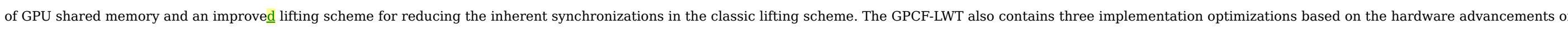

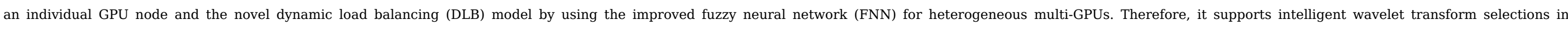
accordance with different surface characterization requirements and measurement conditions.

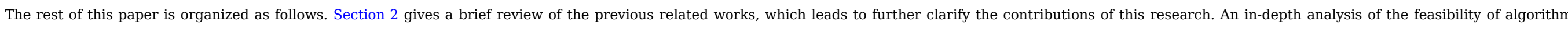

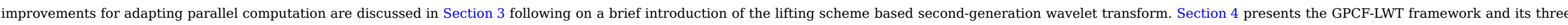

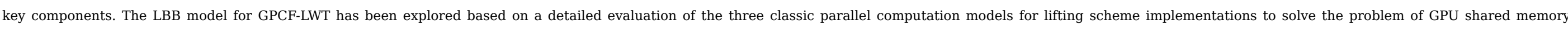

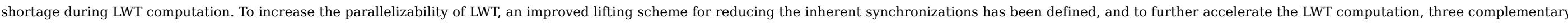

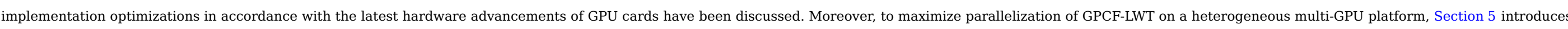

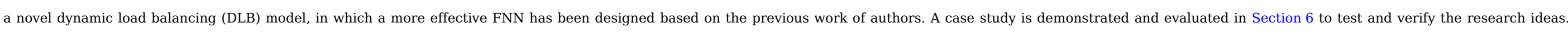
Finally, Section 7 concludes this paper and summarizes the future works.

\section{Previous related works}

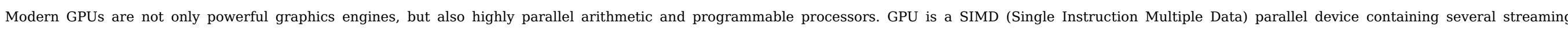

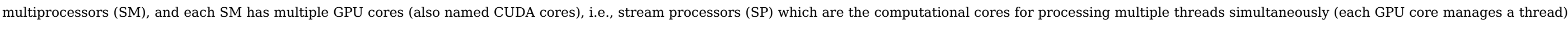

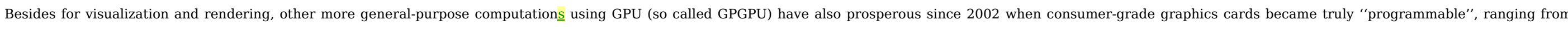

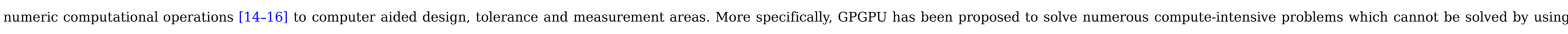

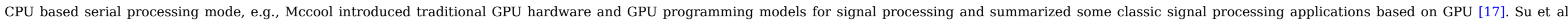




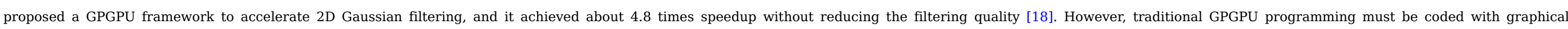

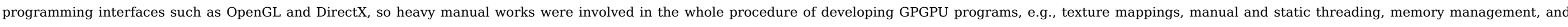
shading program execution. Thus, this obsolete GPGPU programming procedure is very complicated, and it also limited the parallel computing ability of GPU cards.

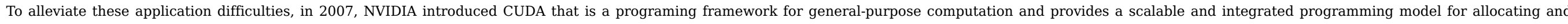

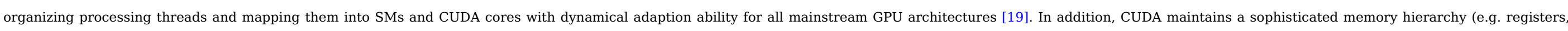

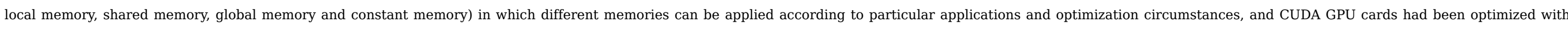

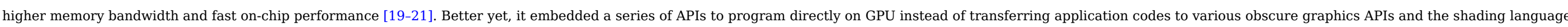

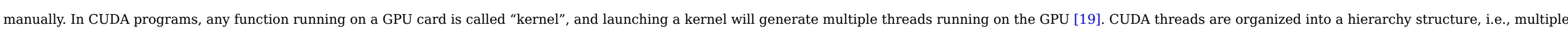

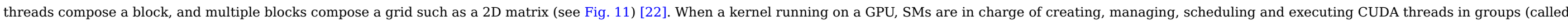

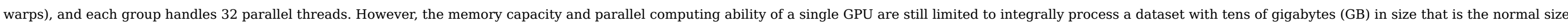

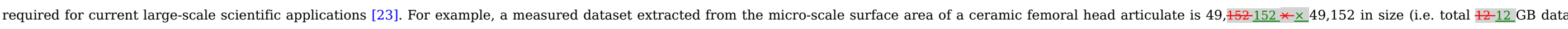

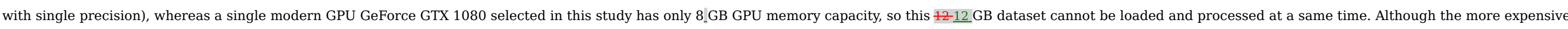

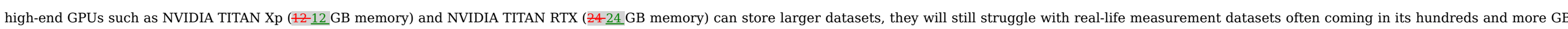

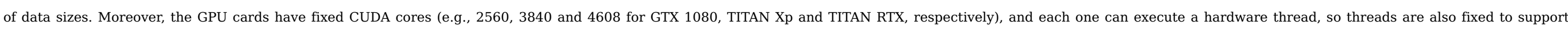

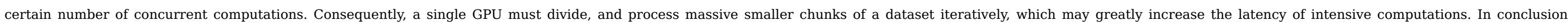

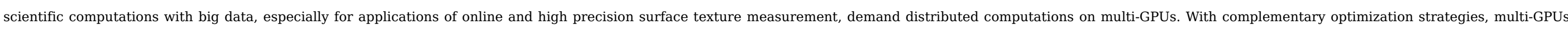

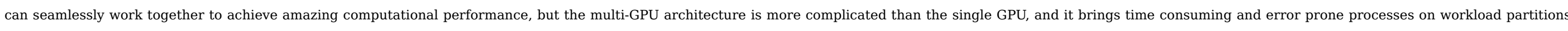

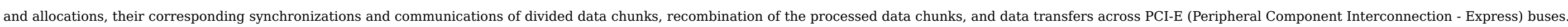

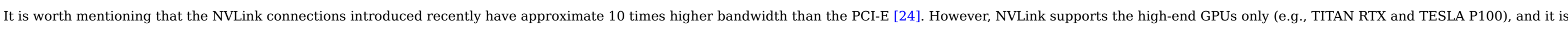
normally equipped only in the supercomputers.

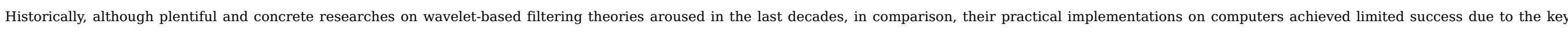

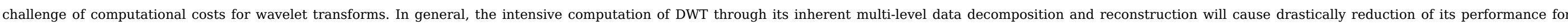

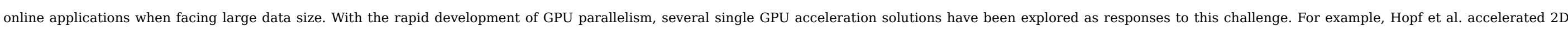

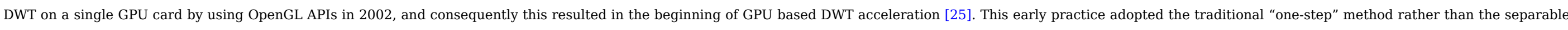

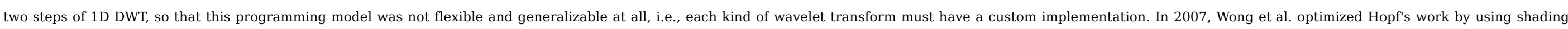

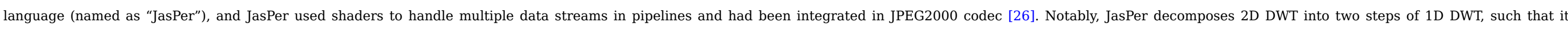

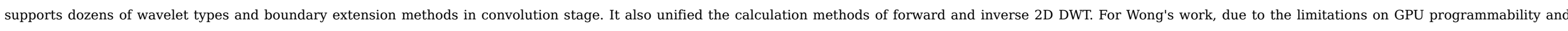

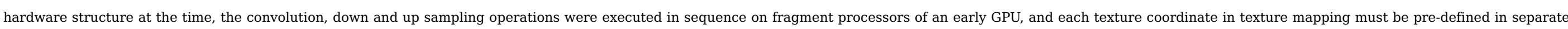

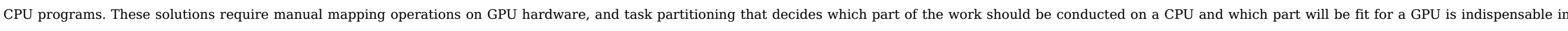
these practices prior to the emerging of unified GPU languages such as CUDA.

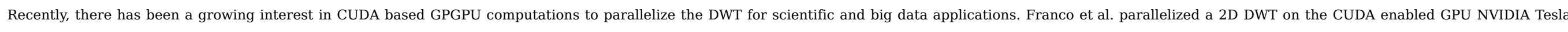

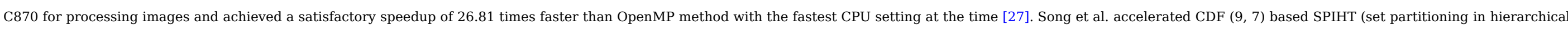

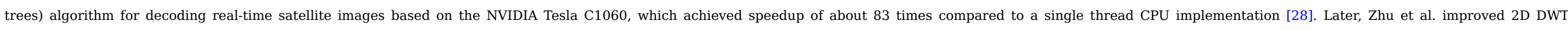

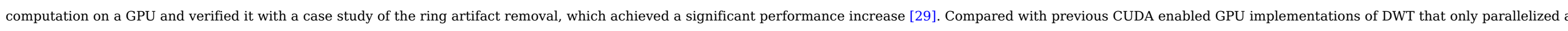

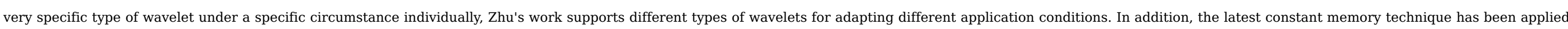

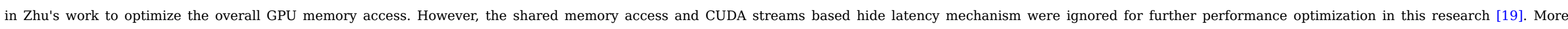

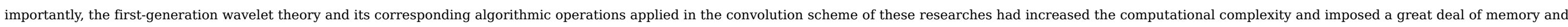




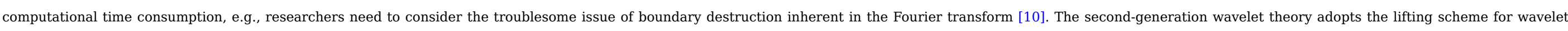

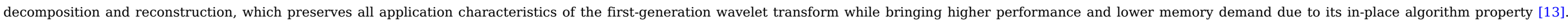

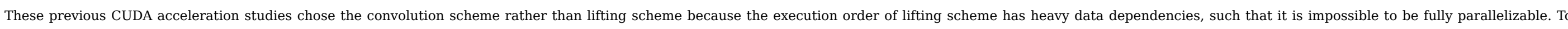

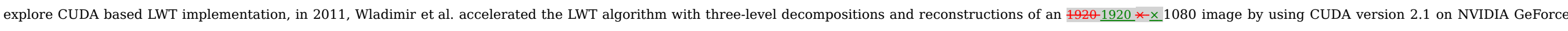

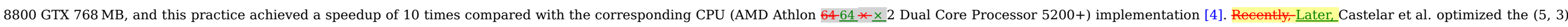

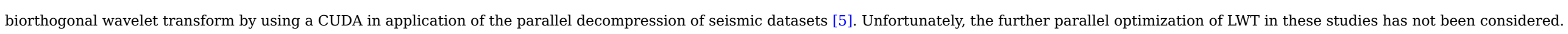

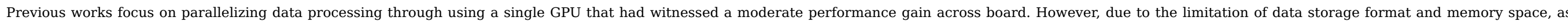

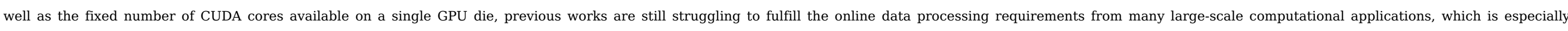

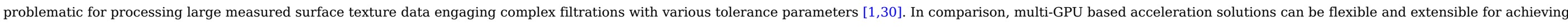

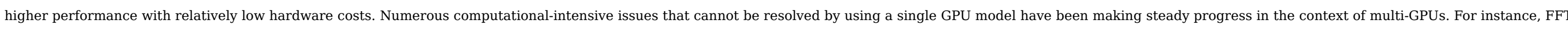

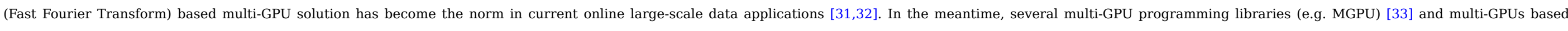

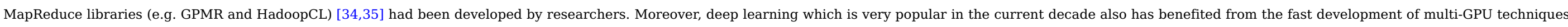

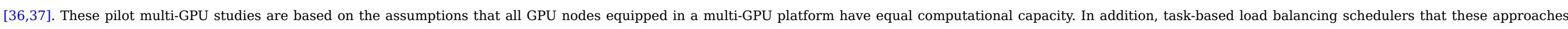

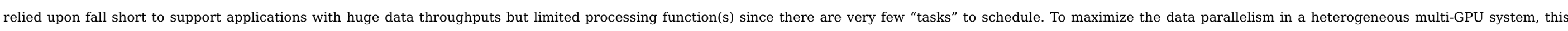
study has also devised an innovative data-oriented dynamic load balancing (DLB) model based on an improved FNN for rapid measured data division and allocation on heterogeneous GPU nodes [38].

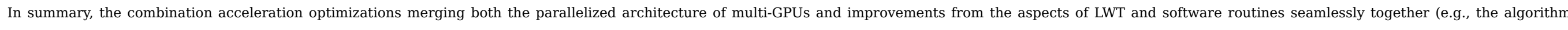

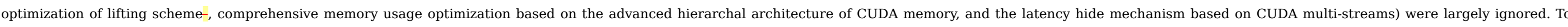

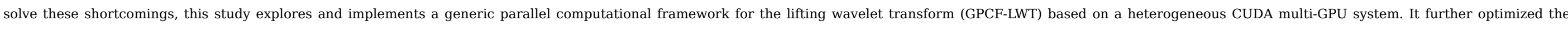

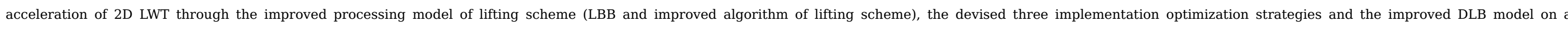

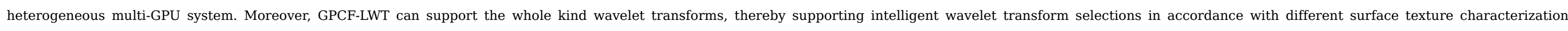
requirements and measurement strategies.

\section{The second-generation wavelet}

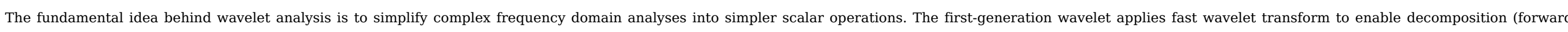

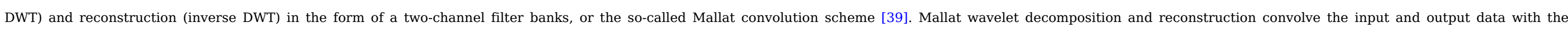

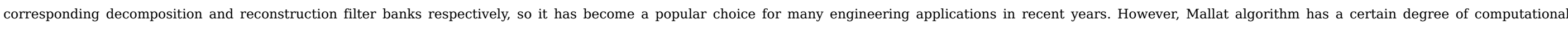
complexity (e.g. it has inherent boundary destruction when executing FFT). Thus, Sweldens proposed a new implementation of DWT, i.e. lifting scheme or the second-generation wavelet.

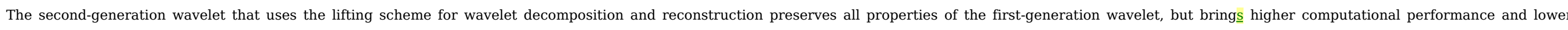

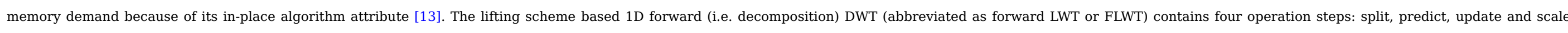
$[4,13,40]$ :

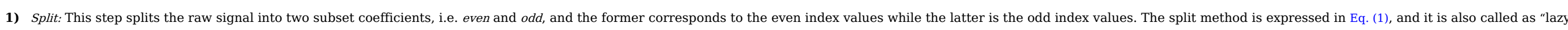
wavelet".

$\{$ even $[i]=X[2 i]$ $\{\operatorname{odd}[i]=X[2 i+1]$

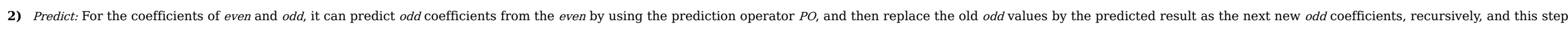




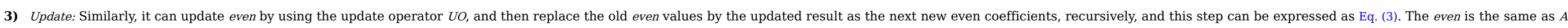
in Mallat algorithm.

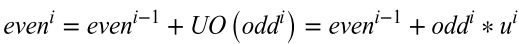

4) Scale: Normalize even and odd coefficients with factor $K$ respectively by using Eq. (4) to get the final approximation coefficients (evenApp) and the detail coefficients (oddDet).

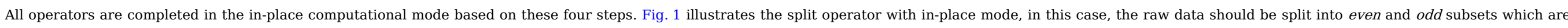

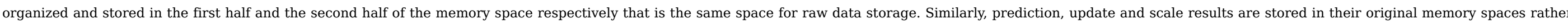

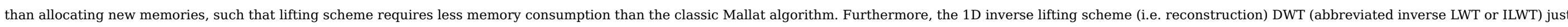
inverse the computational sequence of operation steps of FLWT and switch the corresponding plus and subtraction operators:

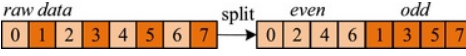

Fig. 1 The computation of split operation with in-place mode.

alt-text: Fig 1

Scale:

$\{$ even $=$ evenAPP $\times K$

odd $=$ oddDet $\times(1 / K)$

Update:

even $=$ even $-U O($ odd $)$

Predict:

$o d d=o d d+P O($ even $)$

Merge: This step is also called as "inverse lazy wavelet", and it can be expressed by:

$\left\{\begin{array}{c}X[2 i]=\text { even }[i] \\ X[2 i+1]=\text { odd }[1]\end{array}\right.$

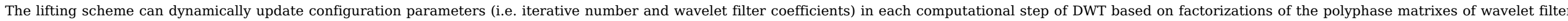

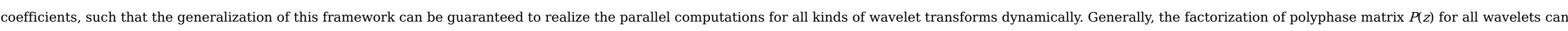
be described as the following:

$P(z)=\prod_{i=1}^{n}\left[\begin{array}{cc}1 & u_{i}(z) \\ 0 & 1\end{array}\right]\left[\begin{array}{cc}1 & 0 \\ p_{i}(z) & 1\end{array}\right]\left[\begin{array}{cc}K & 0 \\ 0 & 1 / K\end{array}\right]$

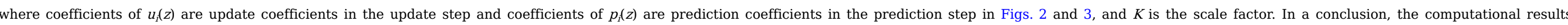




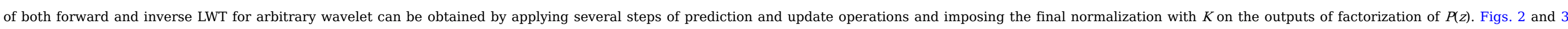
illustrate the main computational processes of single-level 1D forward and inverse LWT respectively.

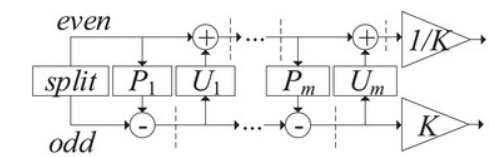

$\cdot P_{i}: i^{\text {th }}$ prediction $\cdot B>$ : normalization with factor $k$

$\cdot U_{i}: i^{\text {lh }}$ update $\quad$ : the synchronization position

Fig. 2 The main computational procedure of single-level 1D forward LWT.

alt-text: Fig 2

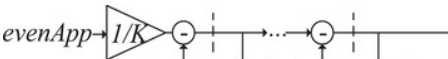

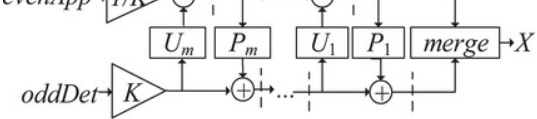

$P_{i}: i^{\text {ih }}$ prediction $\cdot \mathbb{B}$ : normalization with factor $k$

$U_{1}: i^{\text {th }}$ update $\quad \cdot$ : the synchronization position

Fig. 3 The main computational procedure of single-level 1D inverse LWT.

alt-text: Fig 3

\section{CUDA based GPCF-LWT implementation}

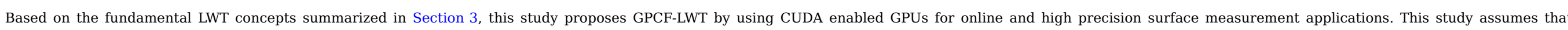

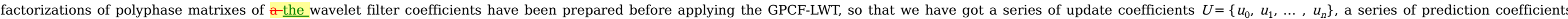
$P=\left\{p_{0}, p_{1}, \ldots, p_{n}\right\}$, and also the scale factor $K$.

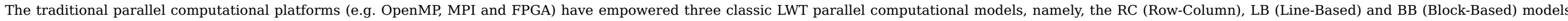

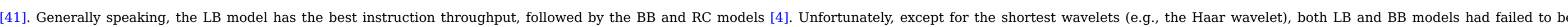

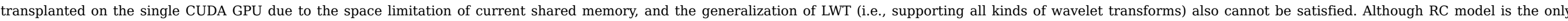

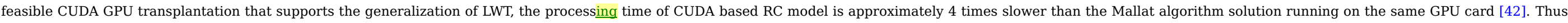
based on the in-depth study of these three models, this study devised a generic LWT parallel computational model for the CUDA enabled GPU architecture.

\subsection{The improved parallel computational model}

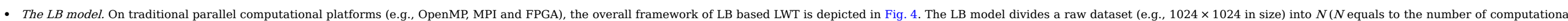
nodes) smaller subsets (e.g., if $N$ is 4 , each subset has 256 rows and 1024 columns), and each subset will be allocated on a computational node where LB performs the following operations:

1) Lunching $m$ threads, and each one performs horizontal 1D LWT for each row of the target subset concurrently (i.e., each thread processes a row of the target subset.) and then the LB caches the temporary results in the local memory (e.g., a RAM-random access memory);

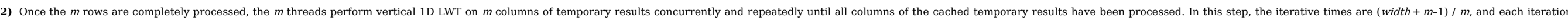
processes $m$ columns.

3) Steps 1 and 2 maintain an integrated iteration that will be repeated until all rows and columns of the target subset are processed. 


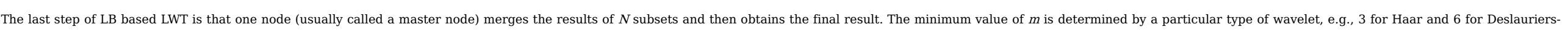

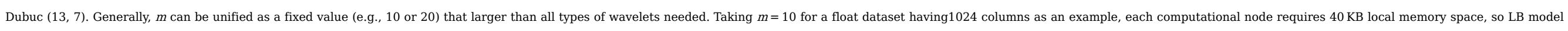

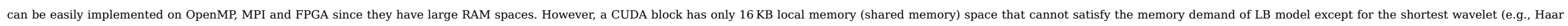
wavelet requires only $12 \mathrm{~KB}$ shared memory space). In addition, columns of a dataset cannot be divided by LB, so it is a coarse-grained division approach, which cannot operate well with the SIMD of CUDA.

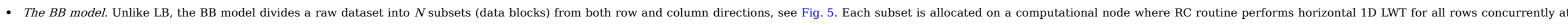

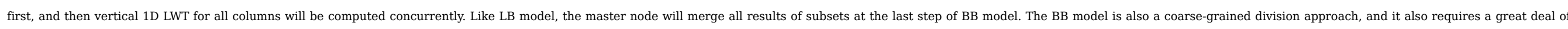

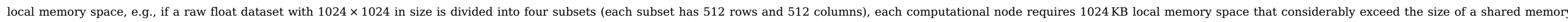
space of a CUDA GPU, such that classic RC model based CUDA solutions must use a GPU global memory space as the temporary caches, and it has become the key bottleneck for data access.

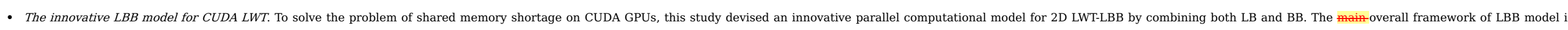

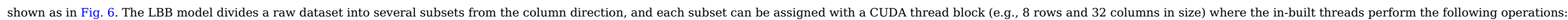

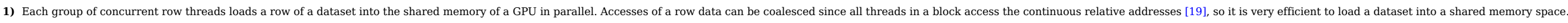

2) Each group of concurrent row threads in a block performs horizontal 1D LWT on a data row in parallel, and then the GPU caches the temporary results in its shared memory space.

3) $m$ groups of row threads get $m$ row temporary results in parallel by executing steps 1 and 2 ( $m$ is a preset value, and it is 8 here)

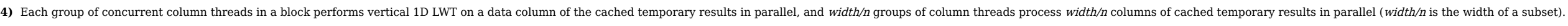

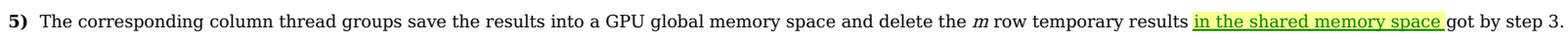

6) Steps 1-5 maintain a complete iteration that will be repeated until all rows and columns of a dataset are processed.

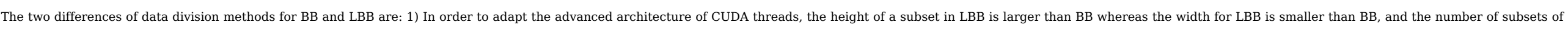

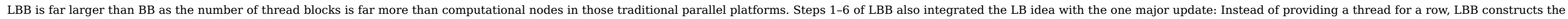

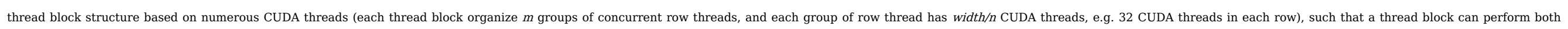
horizontal 1D LWT on multiple rows by using groups of row threads and vertical 1D LWT on multiple columns by using groups of column threads in parallel.

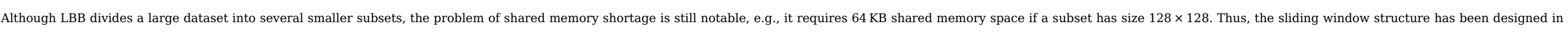

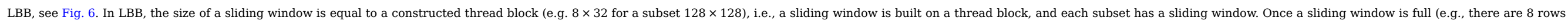

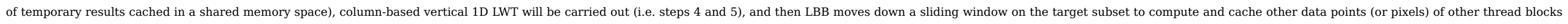
concurrently by moving the throw block to process the next 8 rows (starting from Step 1).

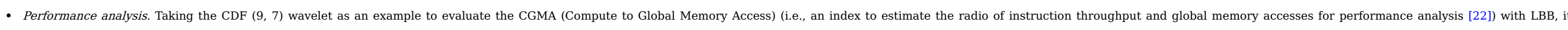

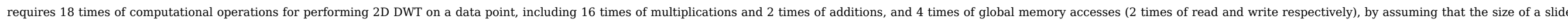
window is $8 \times 32$ and each thread processes a data point in parallel, such that the CGMA is:

$C G M A=C / M=((16+2) \times 2) /(2+2)=9$ 


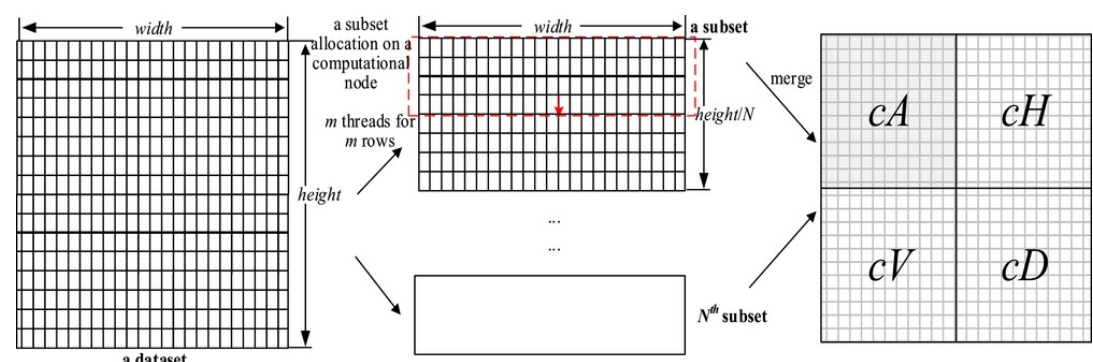

Fig. 4 The overall framework of LB model.

alt-text: Fig 4

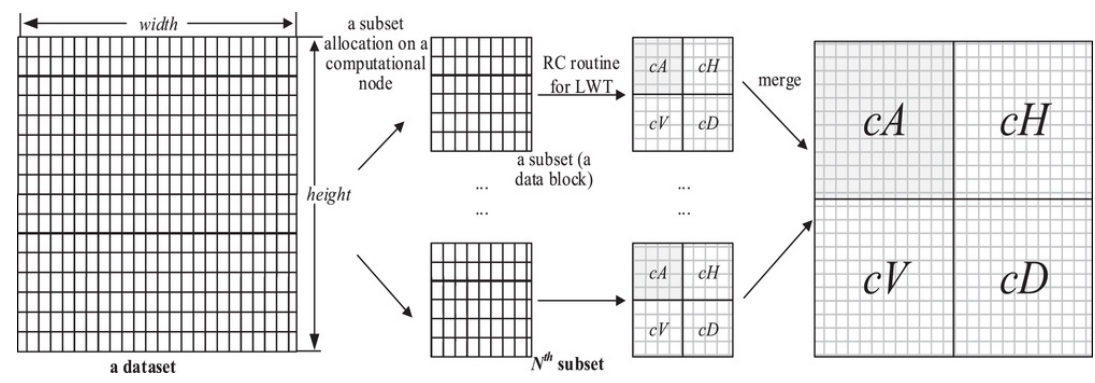

Fig. 5 The overall framework of BB model.

alt-text: Fig 5

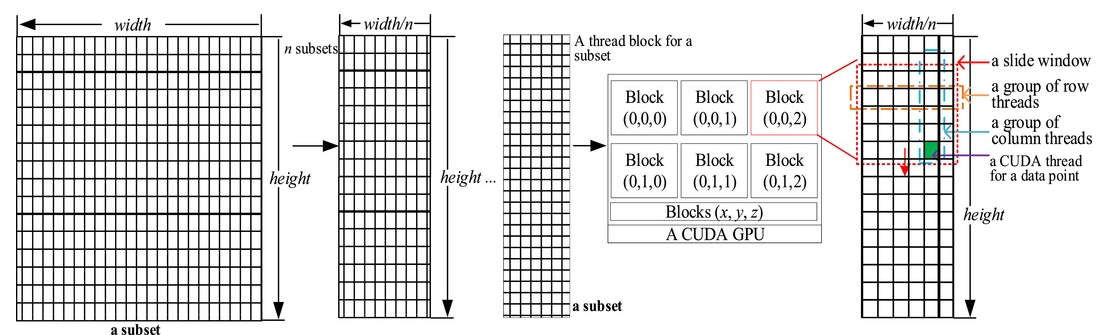

Fig. 6 The overall framework of LBB model.

alt-text: Fig 6

\subsection{The improved lifting scheme}

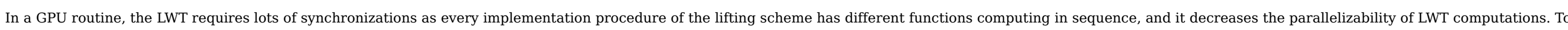

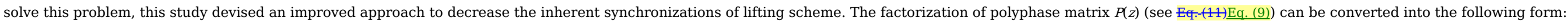

$P(z)$

$$
\prod_{i=0}^{m}\left[\begin{array}{cc}
1 & u_{i}(z) \\
0 & 1
\end{array}\right]\left[\begin{array}{cc}
1 & 0 \\
p_{i}(z) & 1
\end{array}\right]\left[\begin{array}{cc}
K & 0 \\
0 & 1 / K
\end{array}\right]
$$

$=\left[\begin{array}{cc}1 & u_{0}(z) \\ 0 & 1\end{array}\right] \prod_{i=1}^{n}\left[\begin{array}{cc}1 & 0 \\ p_{i}(z) & 1\end{array}\right]\left[\begin{array}{cc}1 & u_{i}(z) \\ 0 & 1\end{array}\right]\left[\begin{array}{cc}K & 0 \\ 0 & 1 / K\end{array}\right]$

where $n=m$ if $u_{0}(z)=0$ and $n=m-1$ if $u_{0}(z) \neq 0$. According to Eq-(13)Eq. (11), the optimized operation steps of the lifting scheme based 1D forward LWT are shown as the follows: 
(2) Preprocessing

$$
\left\{\begin{array}{c}
s^{0}=\text { even }^{0}-u_{0} \times \text { odd } \\
d^{0}=o d d^{0}
\end{array}\right.
$$

(3) Lifting (predict and update): for $i=1, \ldots, n$, then:

$$
\left\{\begin{array}{c}
d^{i}=d^{i-1}-p^{i} \times s^{i-1} \\
s^{i}=s^{i-1}-u^{i} \times d^{i}
\end{array}\right.
$$

(4) Scale:

$\left\{\begin{array}{c}\text { evenApp }=s^{n} / K \\ \text { oddDet }=K d^{n}\end{array}\right.$

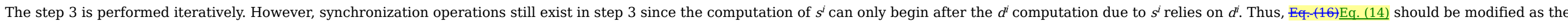
followsing:

$$
\left\{\begin{array} { c } 
{ d ^ { i } = d ^ { i - 1 } - p ^ { i } \times s ^ { i - 1 } } \\
{ s ^ { i } = s ^ { i - 1 } - u ^ { i } \times d ^ { i } }
\end{array} \Rightarrow \left\{\begin{array}{c}
d^{i}=d^{i-1}-p^{i} \times s^{i-1} \\
s^{i}=s^{i-1}-u^{i} \times\left(d^{i-1}-p^{i} \times s^{i-1}\right)
\end{array}\right.\right.
$$

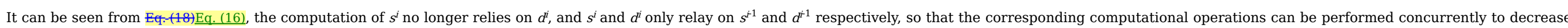

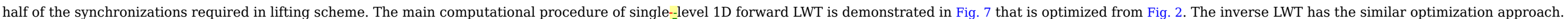
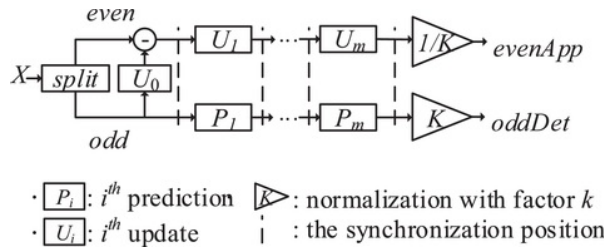

\subsection{The construction of the single GPU-based GPCF-LWT}

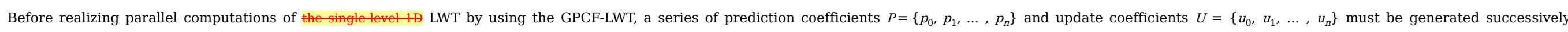

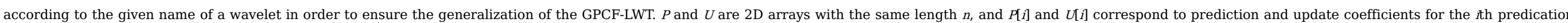

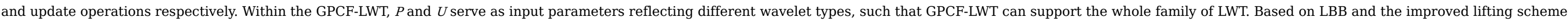

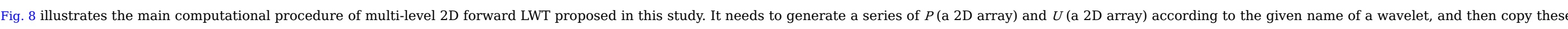

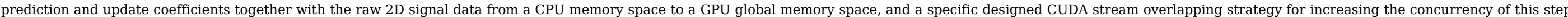




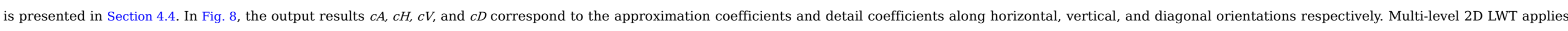

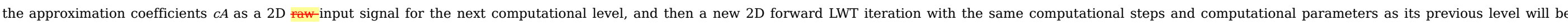

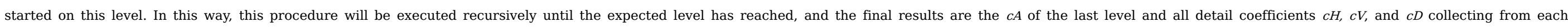
computational level. The computational framework of LBB based inverse LWT can be constructed in the similar way of Fig. 8 .

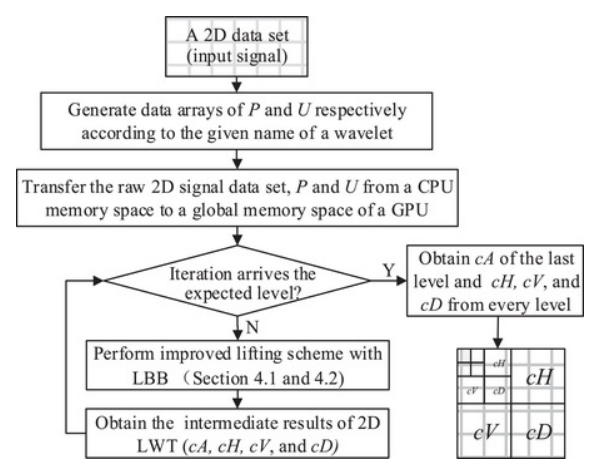

Fig. 8 The main computational procedure of improved lifting scheme with LBB for multi-level 2D forward LWT. alt-text: Fig 8

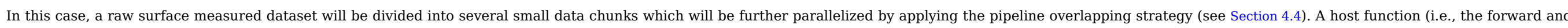

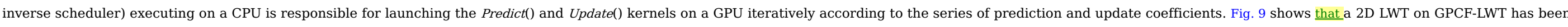

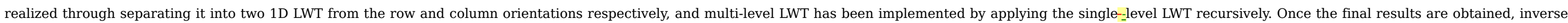
LWT can be performed to reconstruct the filtrated engineering surfaces (e.g. roughness and waviness).

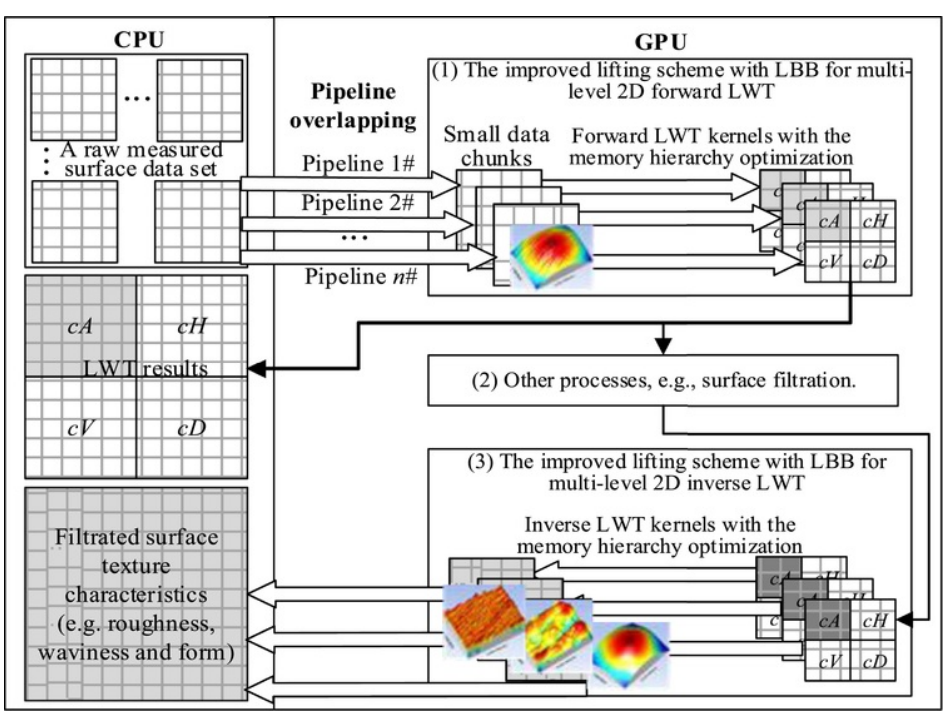

Fig. 9 An implementation overview of the GPCF-LWT on a single GPU card.

alt-text: Fig 9

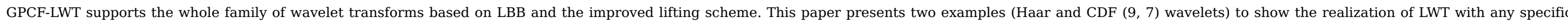




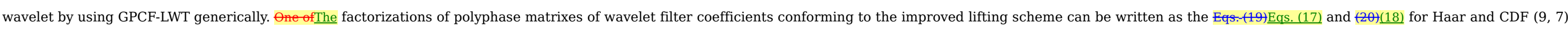
respectively.

$P(z)$

$=\quad\left[\begin{array}{cc}1 & 0 \\ 1 & 1\end{array}\right]\left[\begin{array}{cc}1 & -1 / 2 \\ 0 & 1\end{array}\right]\left[\begin{array}{cc}\sqrt{2} & 0 \\ 0 & 1 / \sqrt{2}\end{array}\right]$

$=\left[\begin{array}{ll}1 & 0 \\ 0 & 1\end{array}\right]\left[\begin{array}{cc}1 & 0 \\ 1 & 1\end{array}\right]\left[\begin{array}{cc}1 & -1 / 2 \\ 0 & 1\end{array}\right]\left[\begin{array}{cc}\sqrt{2} & 0 \\ 0 & 1 / \sqrt{2}\end{array}\right]$,

$P(z)=\left[\begin{array}{cc}1 & 0 \\ -\alpha(1+z) & 1\end{array}\right]\left[\begin{array}{cc}1 & -\beta\left(1+z^{-1}\right) \\ 0 & 1\end{array}\right]\left[\begin{array}{cc}1 & 0 \\ -\gamma(1+z) & 1\end{array}\right]\left[\begin{array}{cc}1 & -\delta\left(1+z^{1}\right) \\ 0 & 1\end{array}\right]\left[\begin{array}{cc}1 / \varepsilon & 0 \\ 0 & 1\end{array}\right] \quad \quad \begin{gathered}\alpha=1.5861343420 \\ \beta=-0.0529801185\end{gathered}$

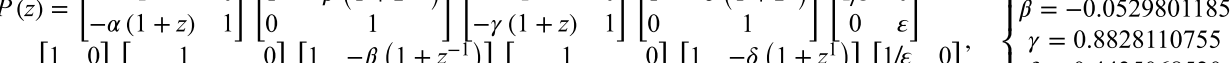

$=\left[\begin{array}{ll}1 & 0 \\ 0 & 1\end{array}\right]\left[\begin{array}{cc}1 & 0 \\ -\alpha(1+z) & 1\end{array}\right]\left[\begin{array}{cc}1 & -\beta\left(1+z^{-1}\right) \\ 0 & 1\end{array}\right]\left[\begin{array}{cc}1 & 0 \\ -\gamma(1+z) & 1\end{array}\right]\left[\begin{array}{cc}1 & -\delta\left(1+z^{-}\right) \\ 0 & 1\end{array}\right]\left[\begin{array}{cc}1 / \varepsilon & 0 \\ 0 & \varepsilon\end{array}\right], \quad\left\{\begin{array}{l}\gamma=0.8828110755 \\ \delta=0.4435068520 \\ \varepsilon=1.1496043988\end{array}\right.$

According to Eqs.(19)Eqs. (17) and (20)(18), $P, U$ and $K$ for Haar and $\operatorname{CDF}(9,7)$ respectively can be acquired from the following Eqs.-(21)Eqs. (19) and (22)(20).

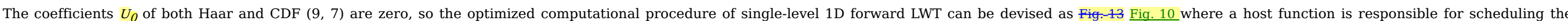

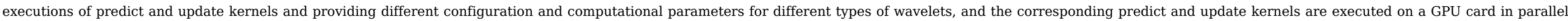

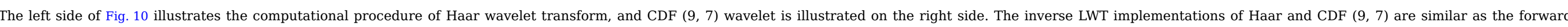
implementations, which just inverse the corresponding sequences of operation steps of forward computations and switch the corresponding plus and subtraction operators.

Harr : $\left\{\begin{array}{c}U_{0}=0 \\ P_{1}=\{1\} \\ U_{1}=\{-1 / 2\} \\ K=1 / \sqrt{2}\end{array}\right.$,

$K=1 / \sqrt{2}$

$\operatorname{CDF}(9,7):\left\{\begin{array}{c}U_{0}=0 \\ P_{1}=\{-\alpha,-\alpha\} \\ U_{1}=\{-\beta,-\beta\} \\ P_{1}=\{-\gamma,-\gamma\} \\ U_{2}=\{-\delta,-\delta\} \\ K=\varepsilon\end{array}\right.$

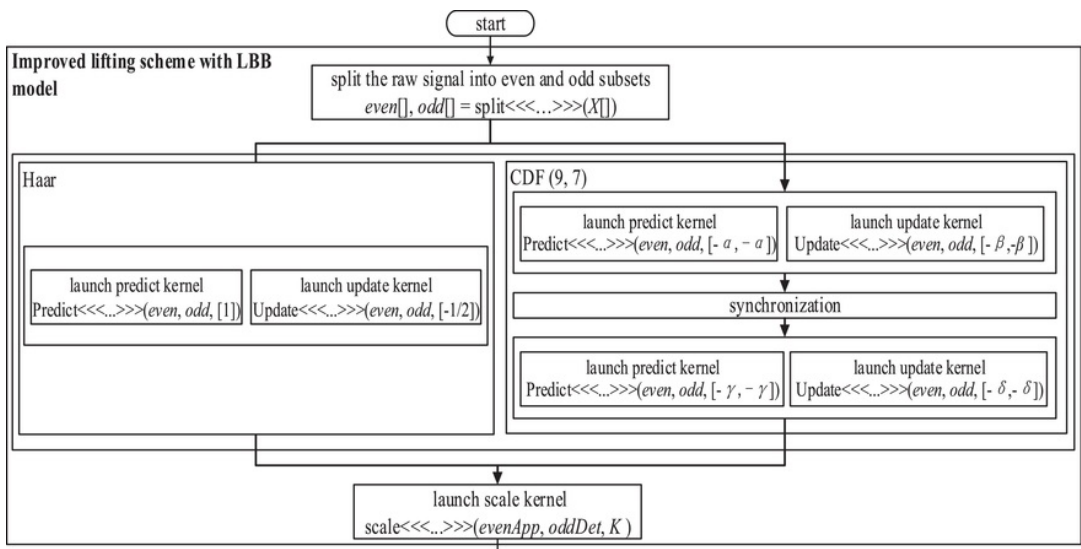

end

Fig. 10 The scheduling algorithm of a single-level 1D forward LWT. 


\subsection{Three optimization strategies for GPCF-LWT implementation on a single GPU card}

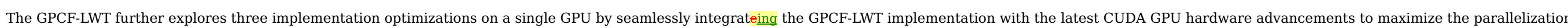
of generic 2D LWT computations:

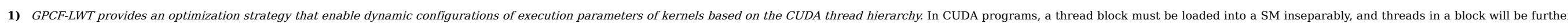

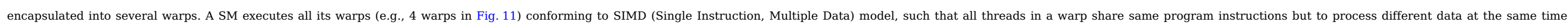

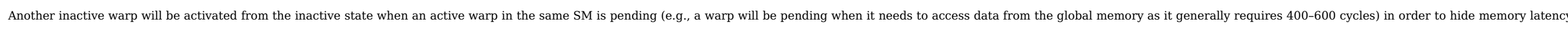

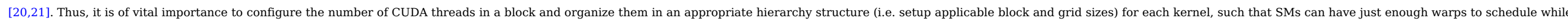

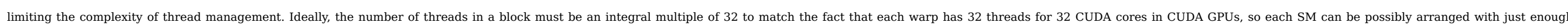

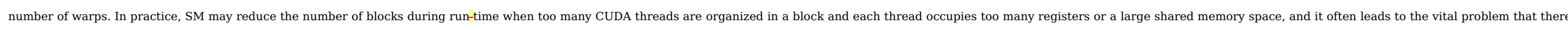

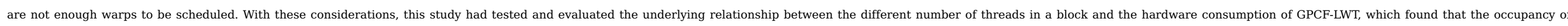

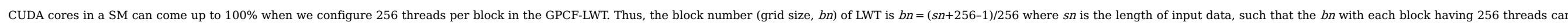

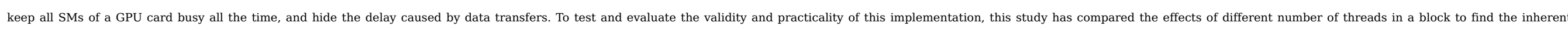

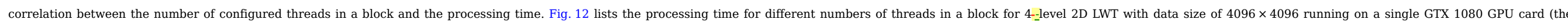
hardware specification can be seen in Table 3). Fig. 12 shows that GPCF-LWT can gain the minimum processing time when the number of threads in a block is set to 256.

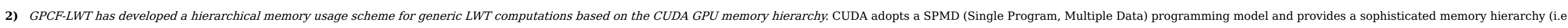

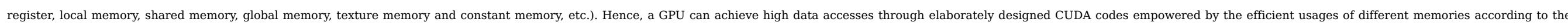

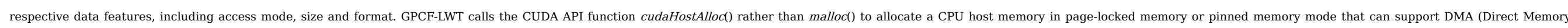

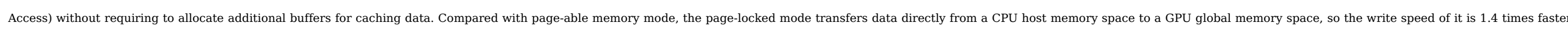

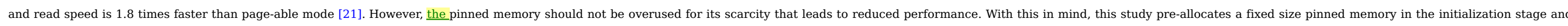

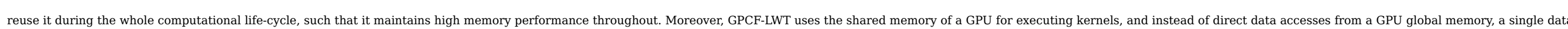

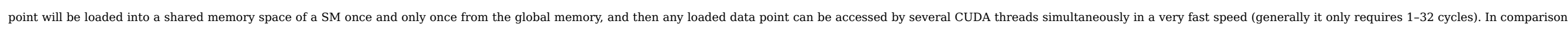

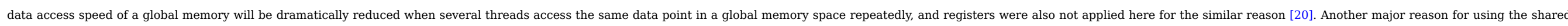

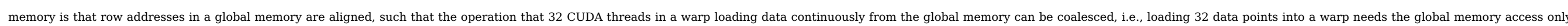

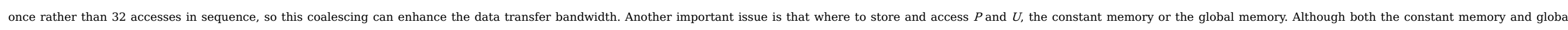

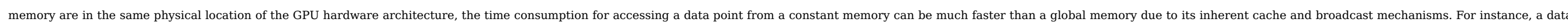

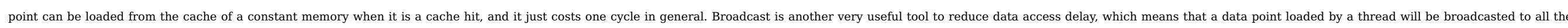

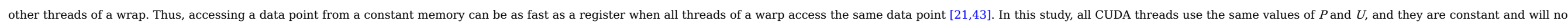

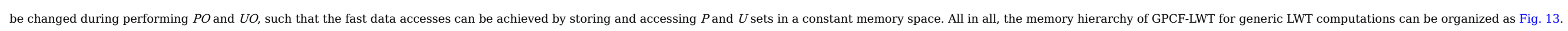

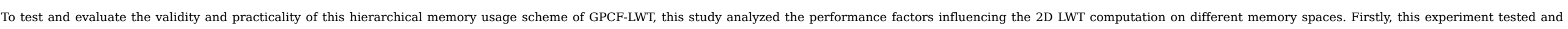

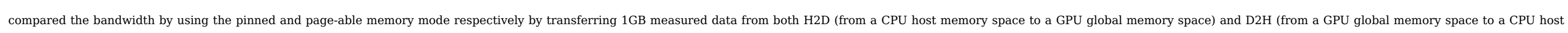

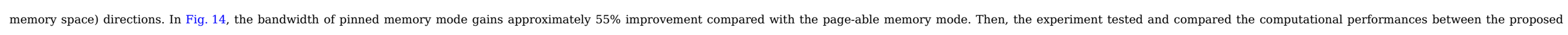

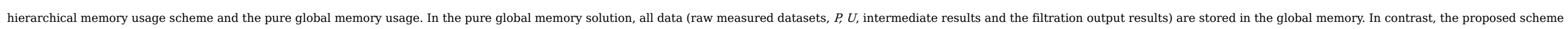

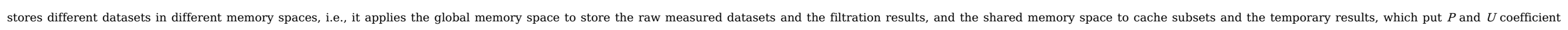
instances in the constant memory space. Fig. 15 illustrates the processing times of these two solutions, and it can be seen from the experimental results that the proposed scheme gains about $50 \%$ computational performance enhancement.

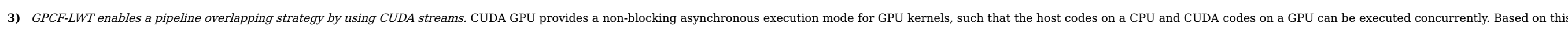




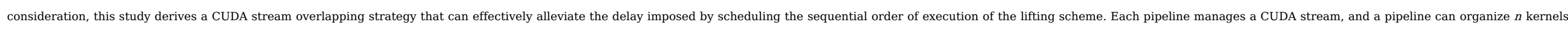

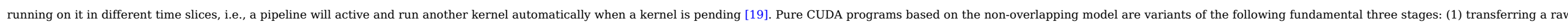

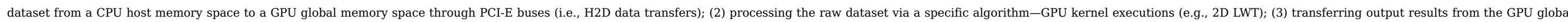

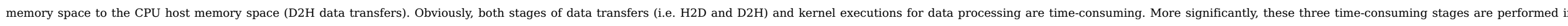

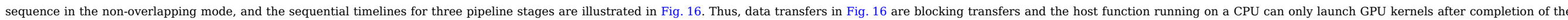

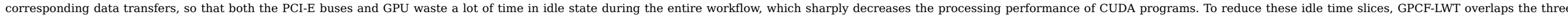

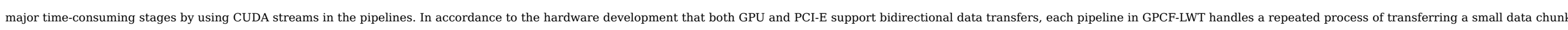

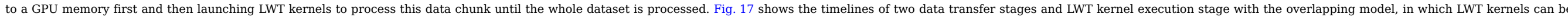

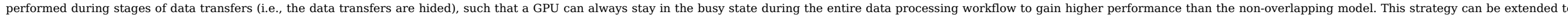

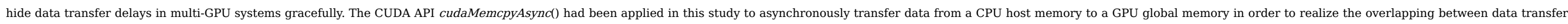

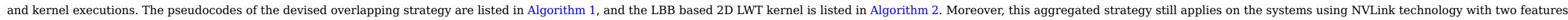

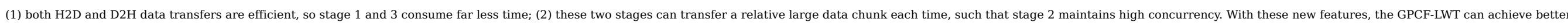
performance without extra effort.

\begin{tabular}{|c|c|c|c|c|}
\hline \multicolumn{2}{|c|}{ Block $(1,0)$} & & \multicolumn{2}{|l|}{\begin{tabular}{|l|} 
Grid \\
\end{tabular}} \\
\hline $\begin{array}{c}\text { Thread } \\
(0,0)\end{array}$ & \begin{tabular}{|c} 
Thread \\
$(0,1)$
\end{tabular} & $\begin{array}{c}\text { Thread } \\
(0, n)\end{array}$ & $\begin{array}{c}\text { Block } \\
(0,0)\end{array}$ & $\begin{array}{c}\text { Block } \\
(0,1)\end{array}$ \\
\hline Thread & Thread & \begin{tabular}{|l|} 
Thread \\
\end{tabular} & $\begin{array}{c}\text { Block } \\
(1,0)\end{array}$ & $\begin{array}{c}\text { Block } \\
(1,1)\end{array}$ \\
\hline
\end{tabular}

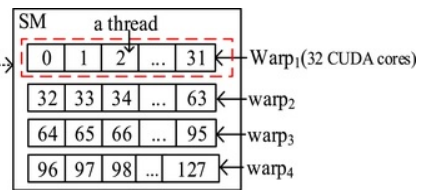

Fig. 11 The hierarchy structure of CUDA threads. alt-text: Fig 11
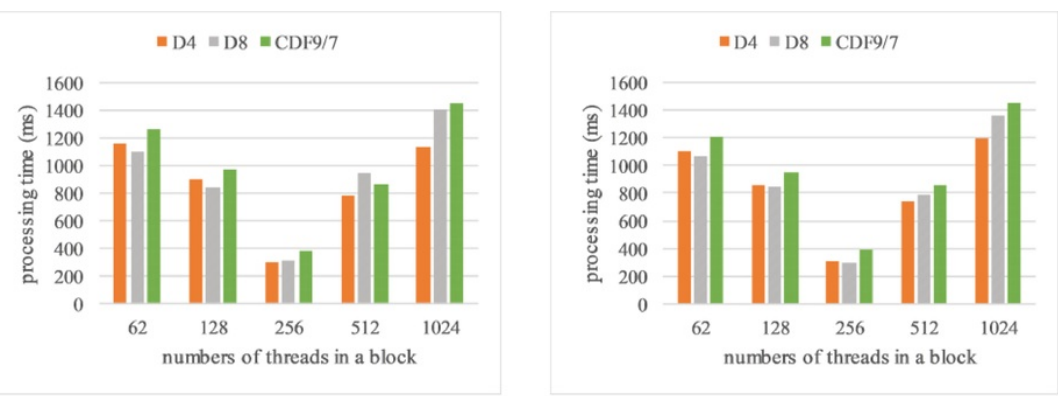

(a) 2D Forward LWT (4 levels)

(b) 2D Inverse LWT (4 levels)

Fig. 12 Processing times of 2D LWT on GPCF-LWT for different numbers of threads in a block.

alt-text: Fig 12 


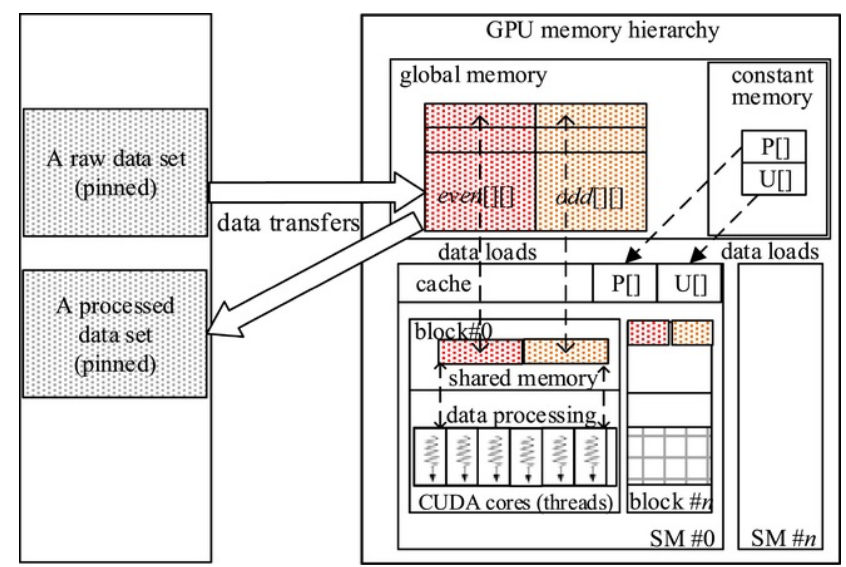

Fig. 13 The hierarchical memory hiorarehy usage of GPCF-LWT.

alt-text: Fig 13

Pinned memory mode \% Page-able memory mode

H2D

D2H

$\begin{array}{llll}0 & 5000 & 10000 & 15000\end{array}$

Fig. 14 The bandwidth of pinned and page-able memory mode respectively.

alt-text: Fig 14

- pure global memory $\%$ hierarchical memory usage schema

LF

ILFT

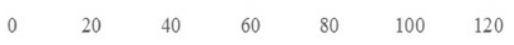

Fig. 15 The performance comparison of the two solutions.

alt-text: Fig 15 


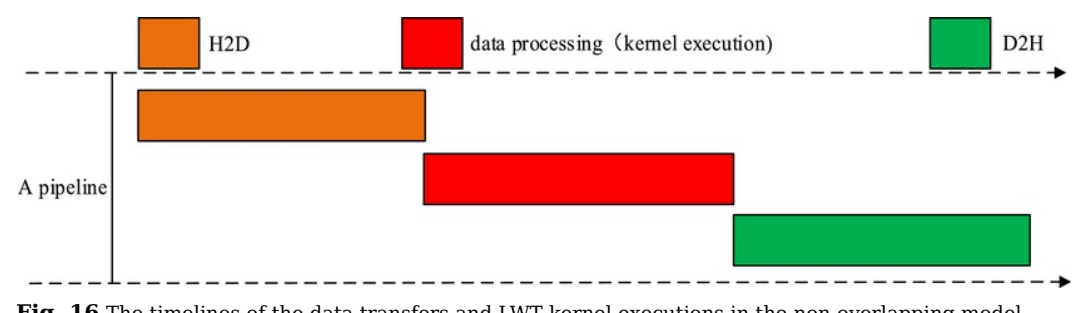

Fig. 16 The timelines of the data transfers and LWT kernel executions in the non-overlapping model.

alt-text: Fig 16

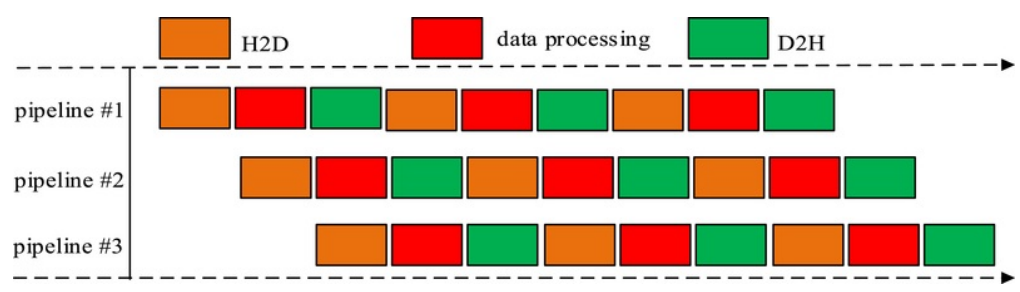

Fig. 17 The timelines for overlapping of three pipelines.

alt-text: Fig 17

Algorithm 1 Overlapping of three stages—two data transfers and kernel executions.

\section{alt-text: Algorithm 1}

Inputs: a 2D raw data Data[][], and row number rows, column number cols.

1 stream <- cudaStreamCreate $(\&$ stream[i]) $\quad / /$ create $n$ streams

$2 a_{-} h=\& D a t a[0][0]$

// get the start address of Data[][]

3 size $=\mathrm{N} *$ cols / nStreams

// calculate the data size for each stream

4 for $i=0$ to $n$ Streams -1 do

5 offset $=i * N /$ nStreams

// calculate the address offset for a stream pipeline

6 cudaMemcpyAsync(a $d+$ offset, a $h+o f f s e t$, size, H2D, stream[i]) // transfer data to a GPU in the th stream

$7 \quad$ LBB_LWT $<<<\ldots$, stream[ $]$ ] $>>>\left(a_{-} d+o f f s e t\right) \quad / /$ launch a wavelet transform kernel in the ith stream

8 cudaMemcpyAsync(a_d+offset, a_h+offset, size, D2H, stream[i]) // transfer data to a CPU in the ith stream

Algorithm 2 LBB based 2D LWT kernel.

alt-text: Algorithm 2

Inputs: a 2D raw data Data[][], and row number rows, column number cols.

Outputs: $c A, c H, c V$, and $c D$.

1 // for each slide window

2 for(row=0; row < height; row $+=$ SLIDE_HEIGHT) do

3 _shared_float sData <- load raw data from a GPU global memory space to a GPU shared memory space

$4 \quad$ // each group of row threads performs horizontal 1D LWT in parallel to process a subset and caches 
// the temporary results

even, odd <- split(sData);

Preprocessing(even, odd);

__syncthreads(); // synchronization operations

predict(even, odd);

update(even, odd);

syncthreads();

// each group of column threads performs vertical 1D LWT in parallel to process the temporary results

even, odd <- split(sData);

Preprocessing(even, odd);

_syncthreads();

predict(even, odd);

update(even, odd);

syncthreads();

save(even, odd, output); // save output results back to a GPU global memory space

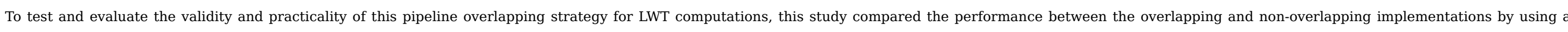

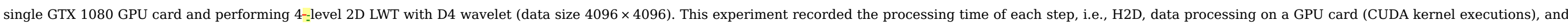

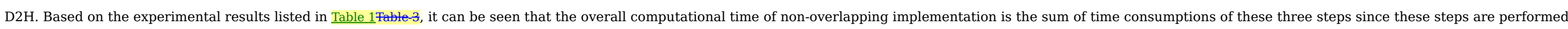

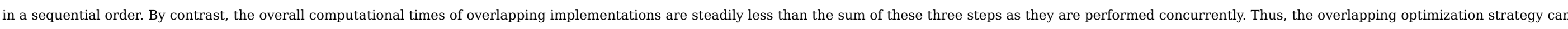
reduce the overall computational time-12.

Table 1 The performance comparison between overlapping and non-overlapping implementation (ms).

alt-text: Table 1

\begin{tabular}{|c|c|c|c|c|c|}
\hline Program routines & Strategies & $\mathrm{H} 2 \mathrm{D}$ & Kernel executions & $\mathrm{D} 2 \mathrm{H}$ & Overall processing time \\
\hline \multirow[t]{2}{*}{ FLWT } & Non-overlapping & 35 & 193 & 32 & 260 \\
\hline & Overlapping & 33 & 197 & 32 & 213 \\
\hline \multirow[t]{2}{*}{ ILWT } & No-overlapping & 33 & 215 & 38 & 286 \\
\hline & Overlapping & 34 & 206 & 35 & 225 \\
\hline
\end{tabular}

\section{The multi-GPU solution of GPCF-LWT}

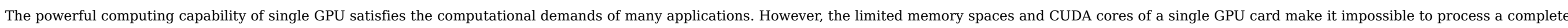

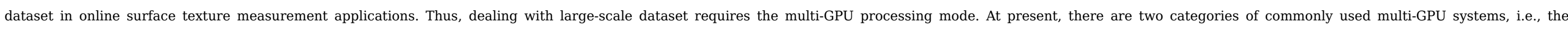

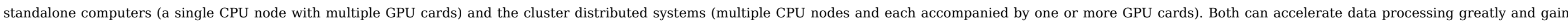

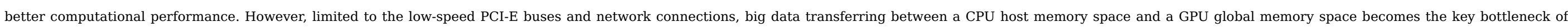

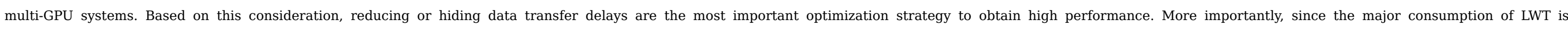




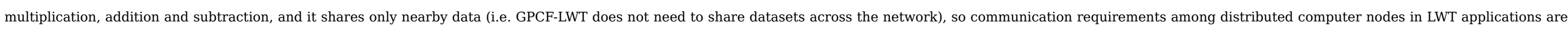

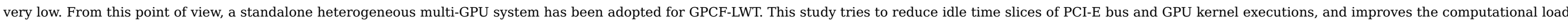

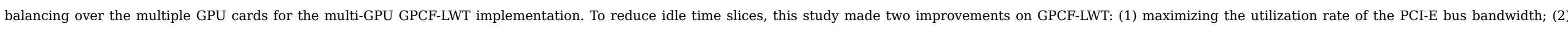

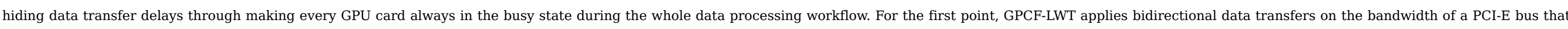

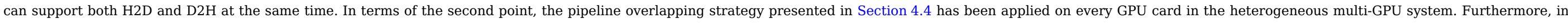

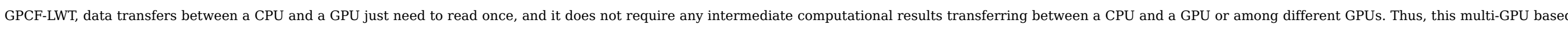

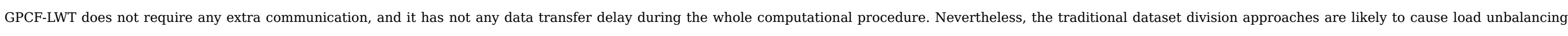
problem and low rate of GPU hardware utilization.

\subsection{Load unbalancing problem}

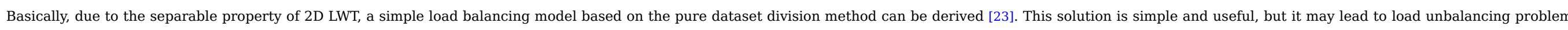

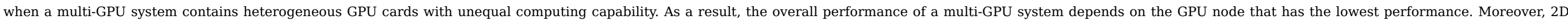

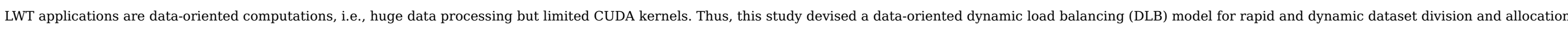

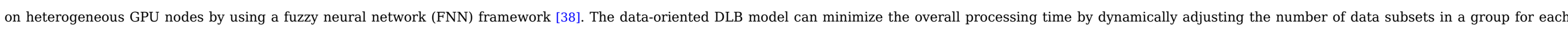
GPU node according to runtime feedbacks. Here, two important improvements on FNN of the previous work of authors ([398]) to enhance the effectiveness of DLB model are presented.

\subsection{Improved FNN model}

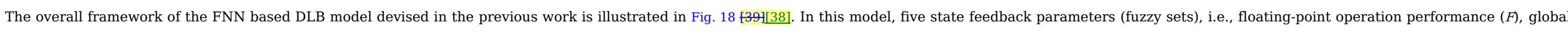

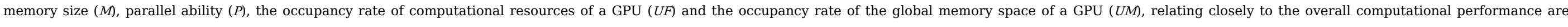

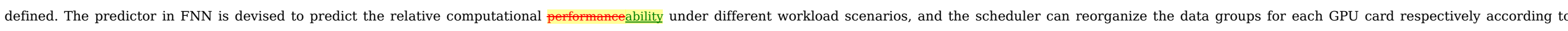

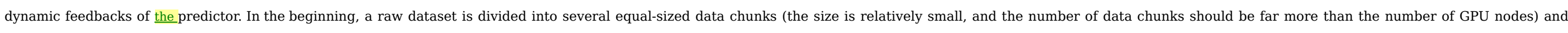

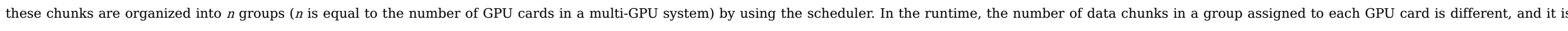

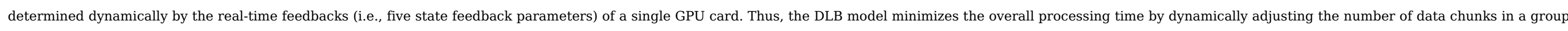
for each GPU at runtime.

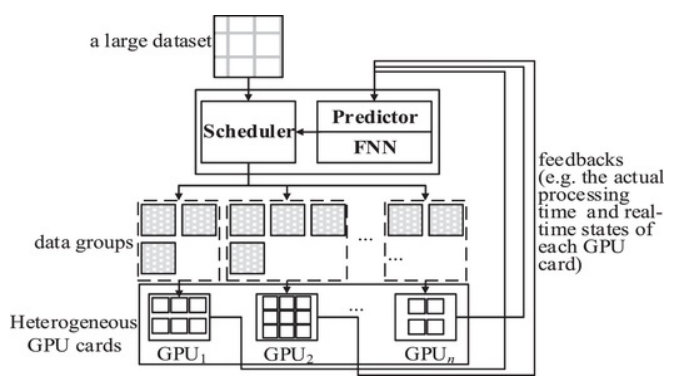

Fig. 18 The overall framework of the FNN based DLB model.

\section{alt-text: Fig 18}

Based on the earlier outputs, this study further explored two improvements on FNN to increase the efficiency and predication accuracy of the devised DLB model:

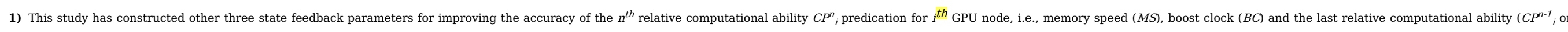

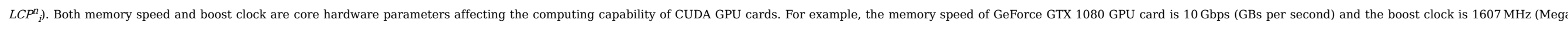




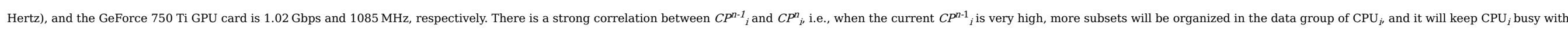

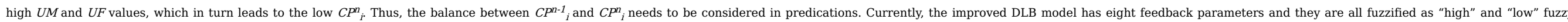
subsets, these three kinds of fuzzy sets and subsets are listed in Table 2.

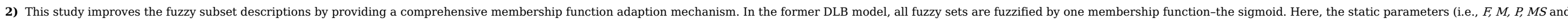

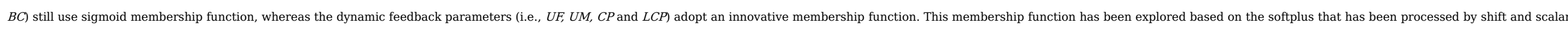

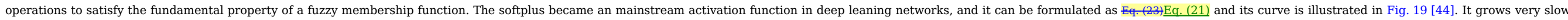

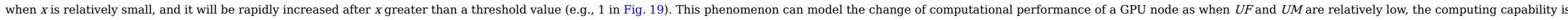
almost unchanged, and it also will drop sharply when $U F$ and $U M$ are larger than the threshold values. However, the softplus cannot be used as a membership function directly due to $E q .(23) E q$. (21) fails to satisfy $f(x) \in[0,1]$ and $x \in[0,1]$.

Table 2 Three kinds of fuzzy sets and subsets.

\section{alt-text: Table 2}

\begin{tabular}{|c|c|c|c|}
\hline Sets & Descriptions & Fuzzy subsets & Descriptions of fuzzy subsets \\
\hline \multirow{2}{*}{ MS } & \multirow{2}{*}{ Memory speed } & $M S L$ & Low \\
\hline & & MSH & High \\
\hline \multirow{2}{*}{$B C$} & \multirow{2}{*}{ Boost clock } & $B C L$ & Low \\
\hline & & $B C H$ & High \\
\hline \multirow{2}{*}{$L C P$} & \multirow{2}{*}{ The last relative computational ability } & $L C P L$ & Low \\
\hline & & $L C P H$ & High \\
\hline
\end{tabular}

Table 3 The specification of a heterogeneous multi-GPU system.

alt-text: Table 3

\begin{tabular}{|c|c|}
\hline Property & Description \\
\hline PU & Intel Core i7-4790 3.6 GHZ \\
\hline Iemory & $16 \mathrm{G}$ \\
\hline \multirow[t]{2}{*}{ PU } & $G P U_{1}$ : NVIDIA GeForce GTX 750 Ti, 2 G, $5 \times$ SM, $128 \mathrm{SP} / \mathrm{SM}, 1.02$ Gbps memory speed, $1085 \mathrm{MHz}$ boost clock \\
\hline & $3 \times G P U_{2}:$ NVIDIA GeForce GTX 1080, 8 G, $20 \times$ SM, 128 SP/SM, 10 Gbps memory speed, $1607 \mathrm{MHz}$ boost clock \\
\hline$S$ & Windows 1064 bit \\
\hline UDA & Version 8.0 \\
\hline
\end{tabular}




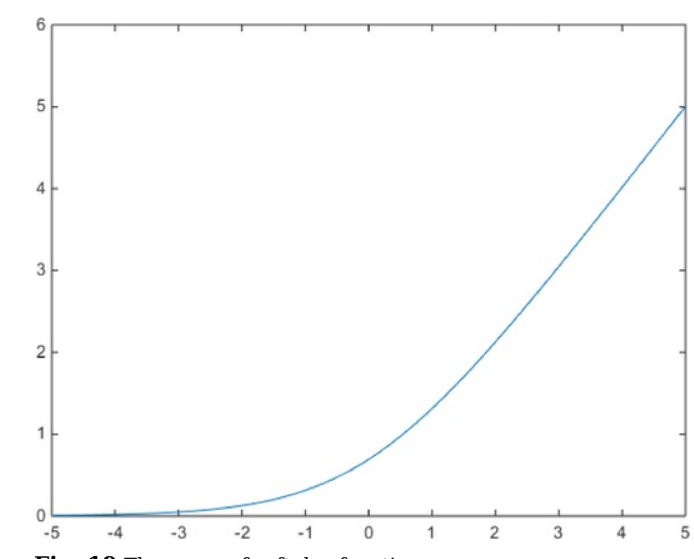

Fig. 19 The curve of softplus function.

alt-text: Fig 19

To remedy this defect, this study performs shift and scalar operations on the softplus function, so the softplus can be extended as follows:

$f(x)=\ln \left(1+e^{a x+b}\right)$

where $a$ is a scalar factor and $b$ is a shift factor, and Eq-(24) Eq. (22) must satisfy:

$\left\{\lim _{x \rightarrow 0} f(x)=0\right.$

$\left\{\begin{array}{l}x \rightarrow 1 \\ \lim _{x \rightarrow 1} f(x)=1\end{array}\right.$

By adding a normalization factor, Eq-(24) Eq. (22) can be redefined as Eq. (26)Eq. (24), and $a$ and $b$ can take the value of 5 and -3 , respectively. Fig. 20 shows the curve of the improved softplus $f(x)=\ln \left(1+e^{a x+b}\right) / \ln \left(1+e^{a+b}\right), x \in[0,1]$

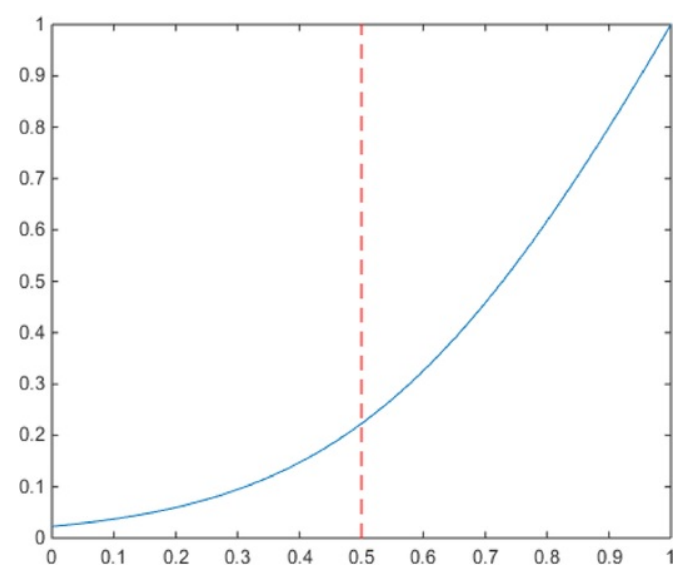

Fig. 20 The curve of the improved softplus function.

alt-text: Fig 20

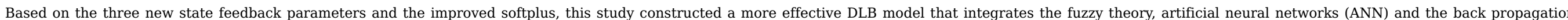
algorithm. The comparison experiment between the improve DLB model and the conventional DLB model in [39] [38] is presented in Section 6.2 (the fourth fifth experiment). 


\section{Test and performance evaluation}

\subsection{Hardware and testbed}

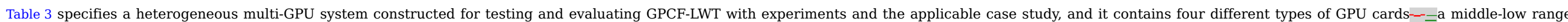
GPU (NVIDIA GeForce GTX 750 Ti) and three high-end GPUs (NVIDIA GeForce GTX 1080). GTX 750 Ti and GTX 1080 contain 640 and 2560 CUDA cores, respectively.

\subsection{Benchmarking experiments}

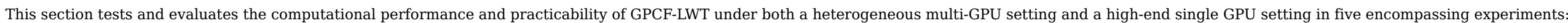

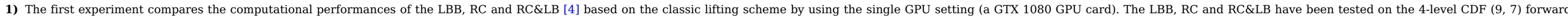

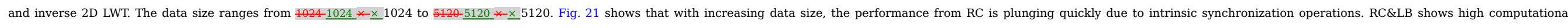

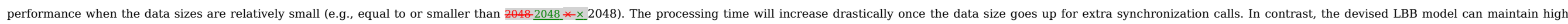

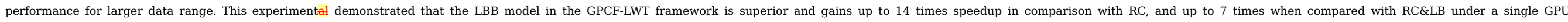
configuration

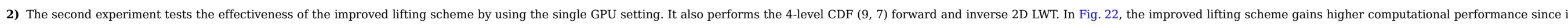
reduces half of the required synchronization in lifting step (step 3) (see Section 4.2). It substantially increases the parallelizability of LWT computations.

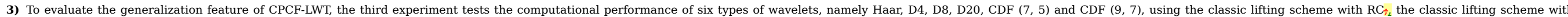

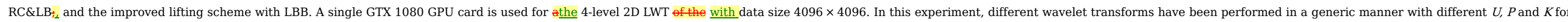
different wavelets. The experimental results highlight that the computational performance of CPCF-LWT is significantly higher than the corresponding RC and RC\&LB based implementations, see Fig. 23 .

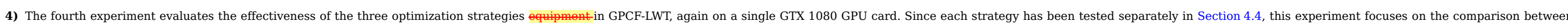
the LBB with the hybrid strategy-driven (the combination of three optimizations) and LBB without any optimization. Fig. 24 shows that the hybrid approach gain noticeable improvements when comparing to the pure LBB.

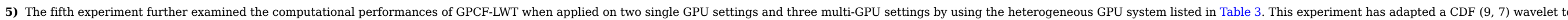

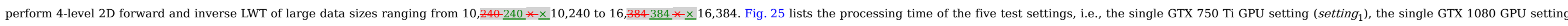

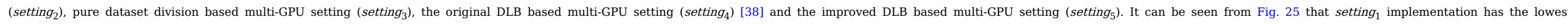

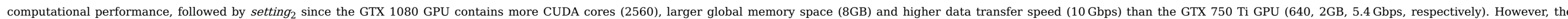

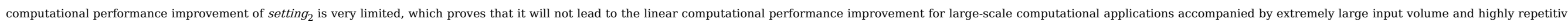

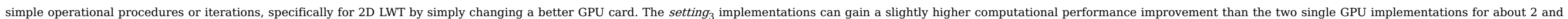

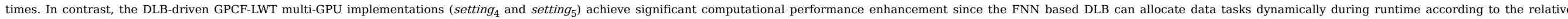

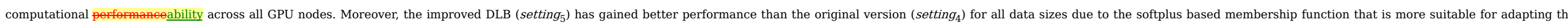

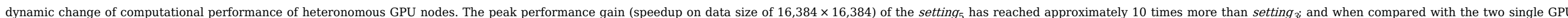
implementations, the speedup from setting $g_{5}$ has reached 15 and 20 times than setting $g_{1}$ and setting $g_{2}$ respectively. 


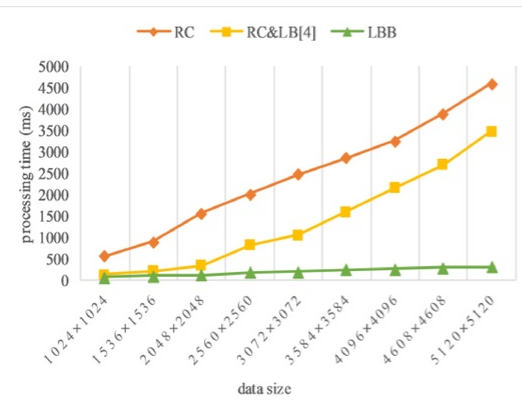

(a) 2D forward LWT

Fig. 21 The performance comparison among LBB, RC and RC\&LB models.

alt-text: Fig 21

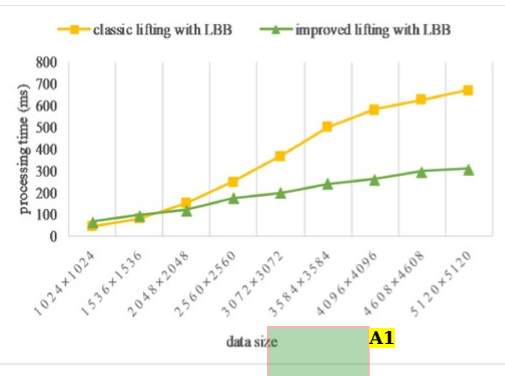

(a) 2D forward 2D LWT

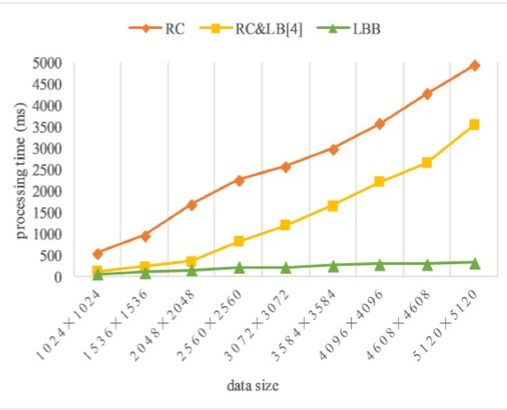

(b) 2D inverse LWT

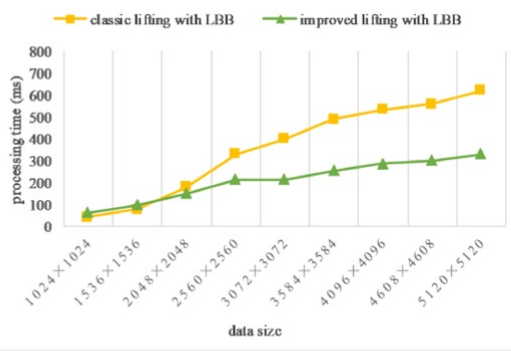

(b) 2D inverse LWT

$$
\text { lifing with LBB }
$$

alt-text: Fig 22

Annotations:

A1. delete the extra "2D"

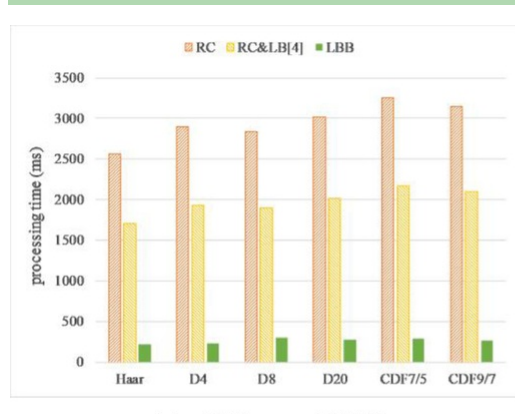

(a) 2D forward LWT 


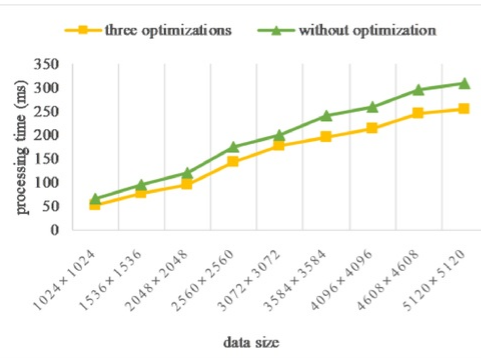

(c) 2D forward LWT

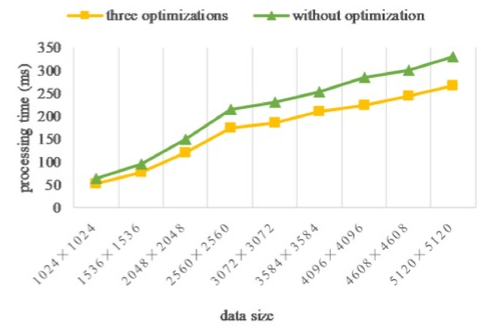

(d) 2D inverse LWT

Fig. 24 The effectiveness experiment of the three optimization strategies in GPCF-LWT.

\section{alt-text: Fig 24}

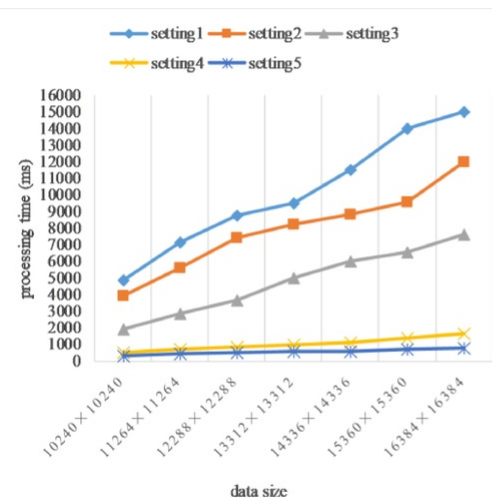

(a) 2D forward LWT

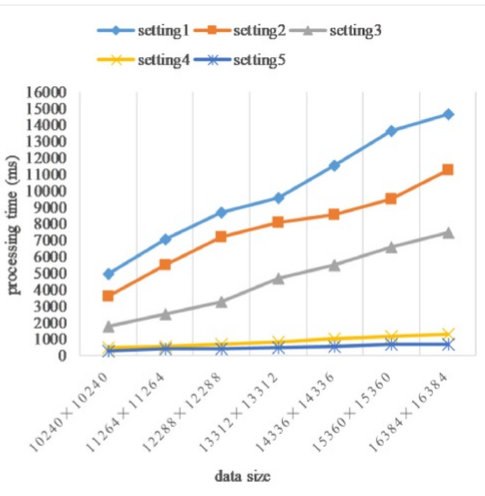

(b) $2 \mathrm{D}$ inverse LWT

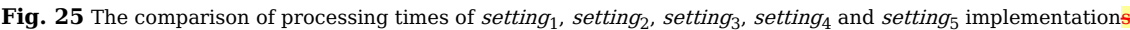

\section{alt-text: Fig 25}

\subsection{A case study on online engineering surface filtration}

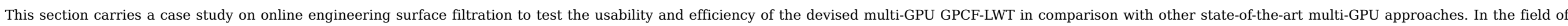

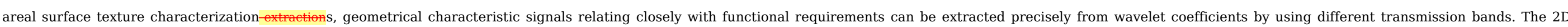

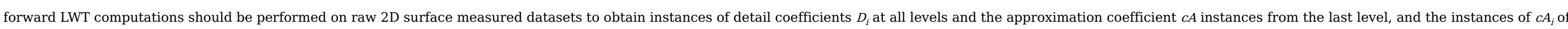

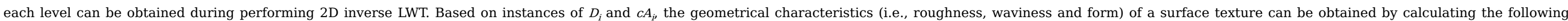
inverse LWT defined in Equation Eq. (27)Eq. (25) [10,40].

$$
\left\{\begin{aligned}
\text { Roughness : } \eta(x, y)=I L W T\left(D_{i, \frac{j}{j^{j-1}}}\right) & =\sum_{i=1}^{l}\left\{D_{i, \frac{j}{j-1}}(x, y)+\rho\left[A_{i-1, \frac{j}{j^{-1}}}(x, y)\right]\right\} \\
\text { Waviness : } \eta^{\prime}(x, y)=\operatorname{ILWT}\left(D_{i, \frac{j}{j^{j-1}}}\right) & =\sum_{i=l}^{i}\left\{D_{i, \frac{j}{j^{j-1}}}(x, y)+\rho\left[A_{i-1, \frac{j}{2^{j-1}}}(x, y)\right]\right\} \\
\text { Form }: \eta^{\prime \prime}(x, y) & =\operatorname{ILWT}\left(A_{i, \frac{j}{j^{j-1}}}\left(x, \frac{k}{2^{j-1}}\right)\right)
\end{aligned}\right.
$$

In order to extract the roughness $\eta(x, y)$ and waviness $\eta^{\prime}(x, y)$ from a surface texture profile, the $c A_{i}$ must be setup to zero. Similarly, the $D_{i}$ must be setup to zero for extracting the form characteristic. 


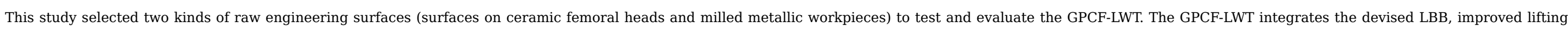

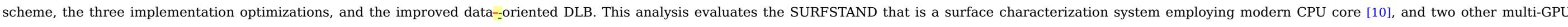

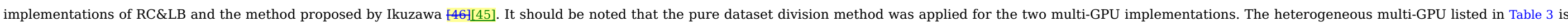
used for all three multi-GPU implementations.

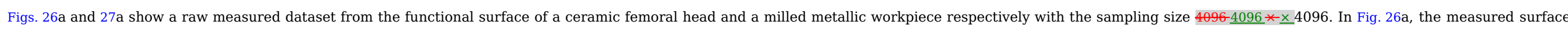

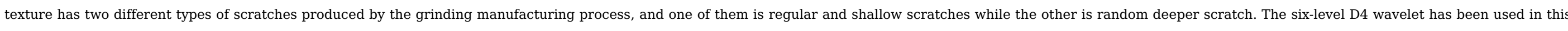

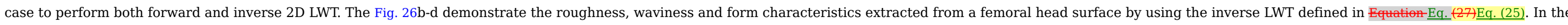

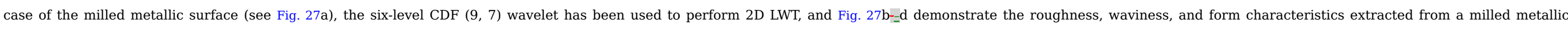
surface by using the inverse LWT defined in Equation Eq. (27)Eq. (25).

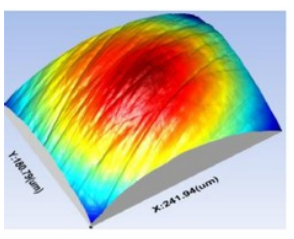

(a) A raw measured surface

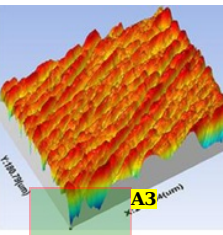

(b) A rough surface

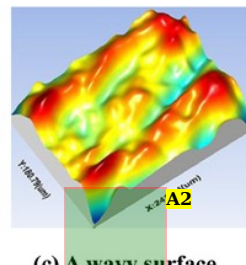

(c) A wavy surface

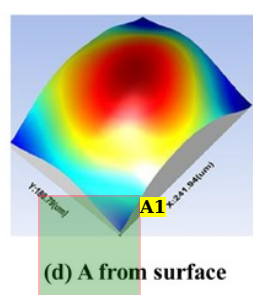

\section{alt-text: Fig 26}

\section{Annotations:}

A1. The form surface

A2. The wavy surface

A3. The rough surface

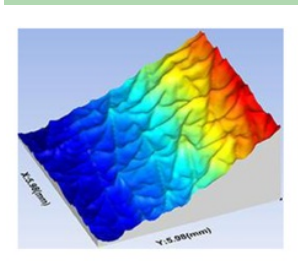

(a) A raw measured surface

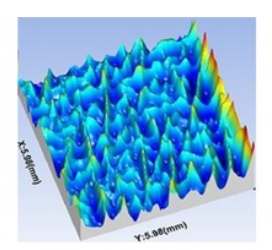

(b) The rough surface

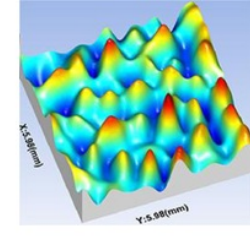

(c) The wavy surface

Fig. 27 The functional surface texture of a milled metallic workpiece.

alt-text: Fig 27

\section{Annotations:}

A1. The form surface

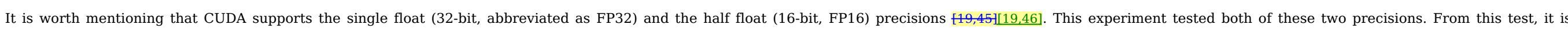

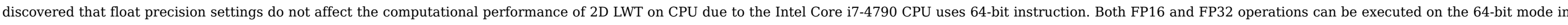

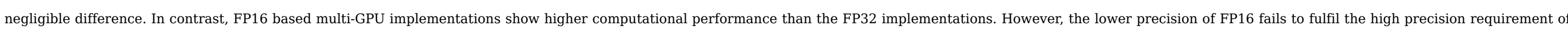

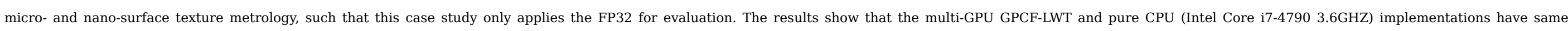
filtration effects on both precision and accuracy aspects. Table 4 shows that multi-GPU implementations can gain over 20 times speedup over CPU implementations. 
Table 4 The performance comparisons between a CPU and the multi-GPU implementation (ms).

alt-text: Table 4

\begin{tabular}{|c|c|c|c|c|}
\hline \multirow[t]{2}{*}{ Datasets } & \multicolumn{2}{|r|}{ LWT } & \multicolumn{2}{|r|}{ ILWT } \\
\hline & CPU & GPCF-LWT & CPU & GPCF-LWT \\
\hline Ceramic femoral head $(4096 \times 4096)$ & 10,165 & 415 & 12,590 & 450 \\
\hline Ceramic femoral head $(12,288 \times 12,288)$ & 28,569 & 756 & 34,681 & 832 \\
\hline Milled metallic workpiece $(4096 \times 4096)$ & 12,850 & 510 & 12,685 & 511 \\
\hline Milled metallic workpiece $(12,288 \times 12,288)$ & 30,256 & 748 & 35,647 & 786 \\
\hline
\end{tabular}

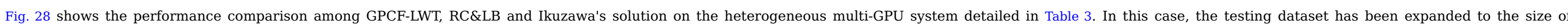

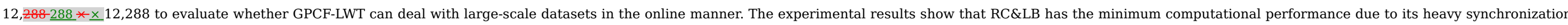

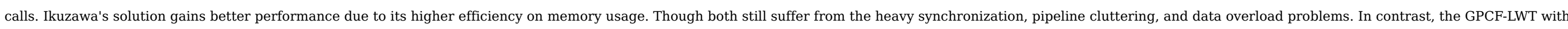
advanced optimizations gains up to 12 times on processing speed over other two multi-GPU approaches.

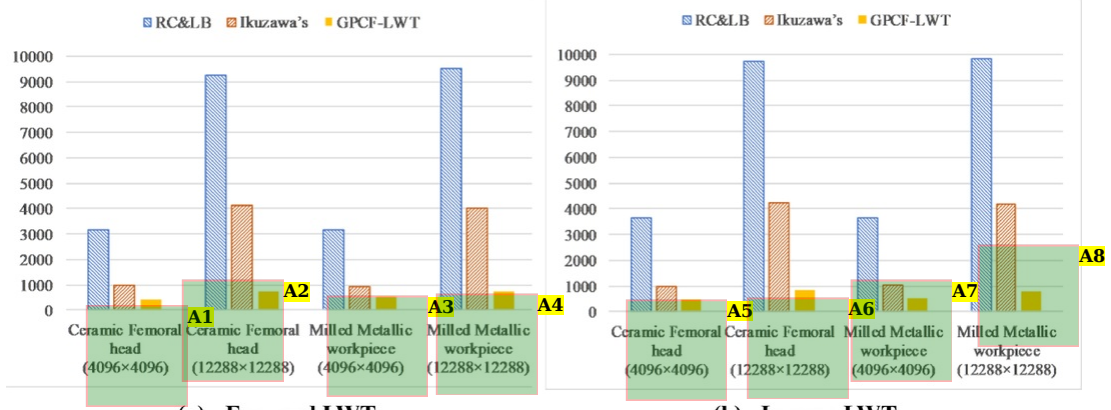

(a) Forward LWT

(b) Inverse LWT

Fig. 28 The performance comparisons of three multi-GPU implementations (ms).

alt-text: Fig 28
Annotations:
A1. Ceramic femoral head
A2. Ceramic femoral head
A3. Milled metallic workpiece
A4. Milled metallic workpiece
A5. Ceramic femoral head
A6. Ceramic femoral head
A7. Milled metallic workpiece
A8. Milled metallic workpiece

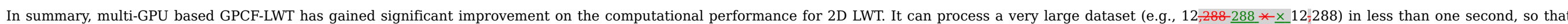
performance requirements of online (or near real-time) and targe data intensive surface texture filtration applications can be satisfied gracefully. 


\section{Conclusions and future work}

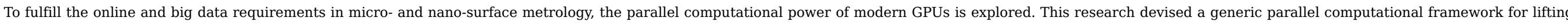

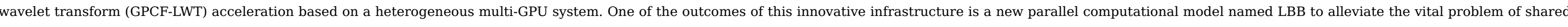

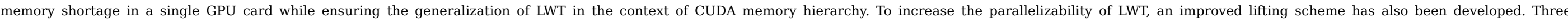

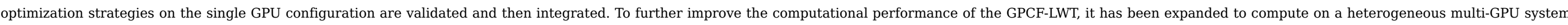

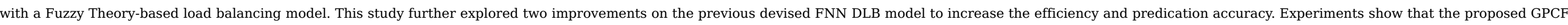

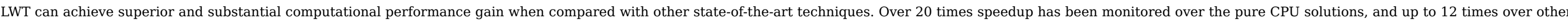

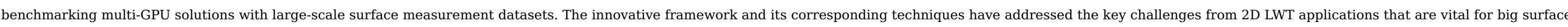
dataset processing

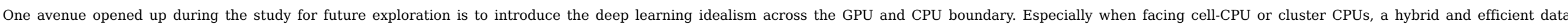
distribution scheme, as well as an online surface texture analysis platform with smart recommendation functions for filter selection will be of great value for practitioners.

\section{Declaration of Competing Interest}

The authors declare that they have no known competing financial interests or personal relationships that could have appeared to influence the work reported in this paper.

\section{Acknowledgments}

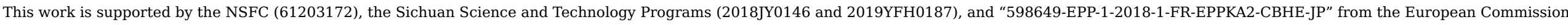

\section{References}

[1] X.J. Jiang and D.J. Whitehouse, Technological shifts in surface metrology, CIRP Ann 61 (2), 2012, 815-836, https://doi.org/10.1016/j.cirp.2012.05.009.

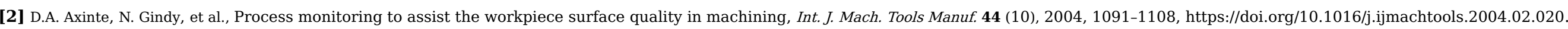

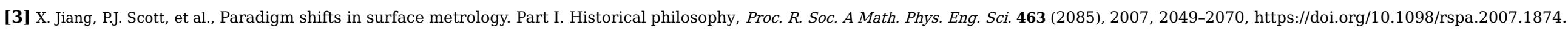

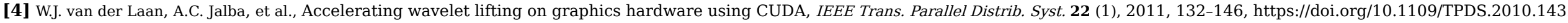

[5] J.A. Castelar, C.A. Angulo, et al., Parallel decompression of seismic data on GPU using a lifting wavelet algorithm, Signal Process. Images Comput. Vis., 2015, IEEE.

[6] S. Lou, W.H.H. Zeng, et al., Robust filtration techniques in geometrical metrology and their comparison, Int. J. Autom. Comput. 10 (1), 2013, 1-8, https://doi.org/10.1007/s11633-013-0690-4.

[7] H.S. Abdul-Rahman, S. Lou, et al., Freeform texture representation and characterisation based on triangular mesh projection techniques, Measurement 92, 2016, 172-182,

https://doi.org/10.1016/j.measurement.2016.06.005.

[8] ISO 16610-61:2015 Geometrical product specification (GPS) - filtration - part 61: linear areal filters - Gaussian filters, (2015).

[9] ASME B46.1-2009, Surface texture: surface roughness, waviness, and lay, (2009).

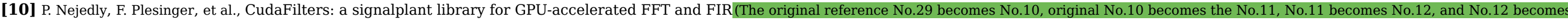

No.13, etc.) filtering, Softw. - Pract. Exp. 48 (1), 2018, 3-9, https://doi.org/10.1002/spe.2507.

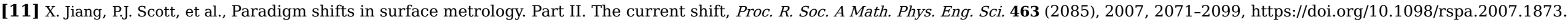

[12] W. Sweldens, The lifting scheme: a construction of second generation wavelets, SIAM J. Math. Anal. 29 (2), 1998, 511-546.

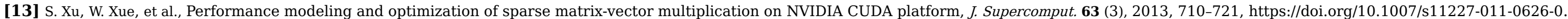


[14] H. Wang, H. Peng, et al., A survey of GPU-based acceleration techniques in MRI reconstructions, Quant. Imaging Med. Surg. 8 (2), 2018, 196-208, https://doi.org/10.21037/qims.2018.03.07.

[15] S. Mittal and J.S. Vetter, A survey of CPU-GPU heterogeneous computing techniques, ACM Comput. Surv. 47 (4), 2015, 1-35, https://doi.org/10.1145/2788396.

[16] M. Mccool, Signal processing and general-purpose computing and GPUs, IEEE Signal Process. Mag. 24 (3), 2007, 109-114, https://doi.org/10.1109/MSP.2007.361608.

[17] Y. Su, Z. Xu, et al., GPGPU-based Gaussian filtering for surface metrological data processing, In: 2008 12th Int. Conf. Inf. Vis., 2008, IEEE, 94-99, https://doi.org/10.1109/IV.2008.14.

[18] NVIDIA, CUDA C Programming Guide v9.0. http://docs.nvidia.com/cuda/cuda-c-programming-guide/.

[19] S. Cook, CUDA Programming: A Developer's Guide to Parallel Computing with GPUs, 2012, Newnes.

[20] NVIDIA, CUDA C Best Practices Guide v9.0, NVIDIA. http://docs.nvidia.com/cuda/cuda-c-best-practices-guide.

[21] D.B. Kirk and W.H. Wen-Mei, Programming Massively Parallel Processors: A Hands-on Approach, 2012, Morgan Kaufmann.

[22] R. Couturier, Designing Scientific Applications On GPUs, 2013, CRC Press.

[23] D. Foley and J. Danskin, Ultra-performance Pascal GPU and NVLink interconnect, IEEE Micro 37 (2), 2017, 7-17, https://doi.org/10.1109/MM.2017.37.

[24] M. Hopf and T. Ertl, Hardware accelerated wavelet transformations, Data Vis., 2000, Springer, 93-103, https://doi.org/10.1007/978-3-7091-6783-0_10.

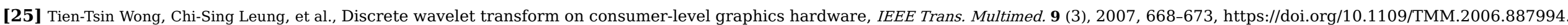

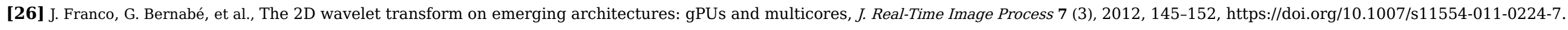

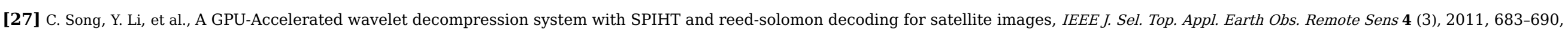
https://doi.org/10.1109/JSTARS.2011.2159962.

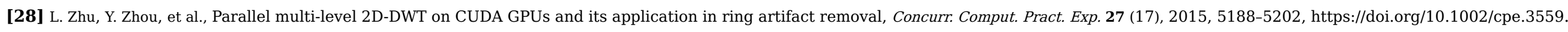

[29] L. Blunt and X. Jiang, Advanced Techniques For Assessment Surface Topography: Development of a Basis For 3D Surface Texture Standards Surfstan 2003, Elsevier,

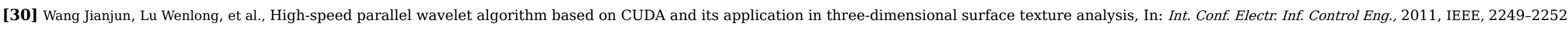
https://doi.org/10.1109/ICEICE.2011.5778225.

[31] Y. Chen, X. Cui, et al., Large-scale FFT on GPU clusters, In: Proc. 24th ACM Int. Conf. Supercomput., New York, New York, USA2010, ACM Presshttps://doi.org/10.1145/1810085.1810128.

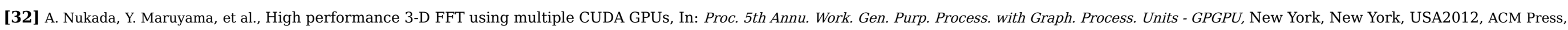
57-63, https://doi.org/10.1145/2159430.2159437.

[33] S. Schaetz and M. Uecker, A multi-GPU programming library for real-time applications, In: Int. Conf. Algorithms Archit. Parallel Process, 2012 , Springer, 114-128.

[34] J.A. Stuart and J.D. Owens, Multi-GPU MapReduce on GPU clusters, In: 2011 IEEE Int. Parallel Distrib. Process. Symp., 2011, IEEE, 1068-1079, https://doi.org/10.1109/IPDPS.2011.102.

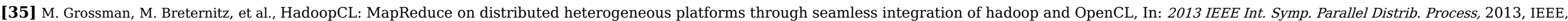
1918-1927, https://doi.org/10.1109/IPDPSW.2013.246.

[36] M. Abadi, A. Agarwal, et al., TensorFlow: large-Scale machine learning on heterogeneous distributed systems, (2016). doi:10.1038/nn.3331.

[37] S. Chintala, An overview of deep learning frameworks and an introduction to Pytorch, 2017. https://smartech.gatech.edu/handle/1853/58786(accessed October 5, 2017) 
[38] C. Zhang, Y. Xu, et al., A fuzzy neural network based dynamic data allocation model on heterogeneous multi-Gpus for large-scale computations, Int. J. Autom. Comput. 15 (2), 2018, 181-193, https://doi.org/10.1007/s11633-018-1120-4.

[39] K.K. Shukla and A.K. Tiwari, Filter banks and DWT, SpringerBriefs Comput. Sci., 2013, Springer, 21-36, https://doi.org/10.1007/978-1-4471-4941-5_2.

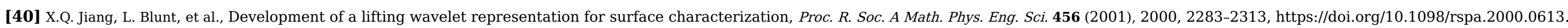

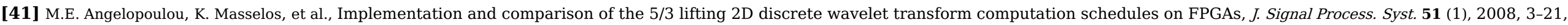
https://doi.org/10.1007/s11265-007-0139-5.

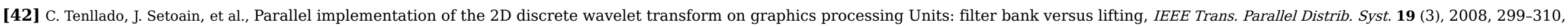
https://doi.org/10.1109/TPDS.2007.70716.

[43] W. Nicholas, The CUDA Handbook: A Comprehensive Guide to GPU Programming, 2013.

[44] Y. LeCun, Y. Bengio, et al., Deep learning, Nature 521 (7553), 2015, 436-444, https://doi.org/10.1038/nature14539.

[45] T. Ikuzawa, F. Ino, et al., Reducing memory usage by the lifting-based discrete wavelet transform with a unified buffer on a GPU, J. Parallel Distrib. Comput. 93-94, 2016, 44-55, https://doi.org/10.1016/j.jpdc.2016.03.010.

[46] NVIDIA, Whitepaper - NVIDIA GeForce GTX 750 Ti, 2014.

\section{Highlights}

- A new parallel computation model for LWT named LBB was devised to tackle the limitation of current shared memory bottleneck on modern GPUs.

- The improved lifting scheme has reduced approximately half of the synchronizations needed when computing the lifting wavelets in a single CUDA thread.

- Three optimization strategies were benchmarked for GPCF-LWT implementation.

- The multi-GPU based GPCF-LWT supports online large-scale measured data filtration through an improved FNN based dynamic load balancing (DLB) model.

\section{Queries and Answers}

Query: Please confirm that givennames and surnames have been identified correctly.

Answer: Yes

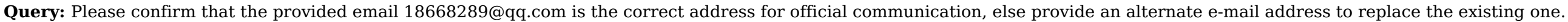
Answer: Please change email address to creekline017@hotmail.com

Query: Please check the citation of Table 1 in the text. It has been put randomly. Please fix it in proper place.

Answer: The citation of Table 1 has been corrected in the text.

Query: Please check the citation of Eq. (26) in the text, But there is no Eq. (26) in the text. Please correct if necessary.

Answer: Eq. (24) is actually Eq. (22), and Eq.26 is actually Eq. (24) . Other wrong equation number had been corrected in text.

Query: Please check the citation of Eq. 27 in the text. It is not in the text. Please correct it if necessary. 
Answer: There are 15 places that the equation number is wrong. We corrected and marked in the text. Thank you.

Query: Please check funding information and confirm its correctness.

Answer: I confirm its correctness

Query: Please provide detail of reference nos. 8 and 9.

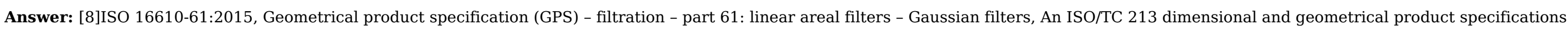
and verification standard, 2015, 1-18, https://www.iso.org/standard/60813.html.

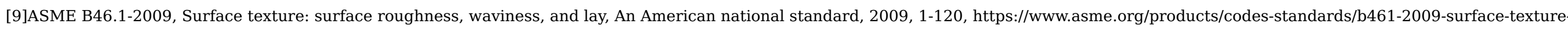
surface-roughness.

Query: Please provide detail of reference no. 36.

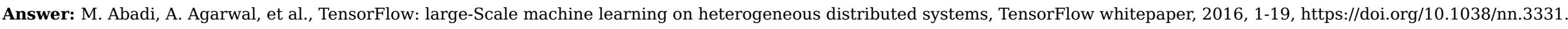

Query: Please provide Publisher name and place for reference no. 45

Answer: No.45 is a journal paper, and You mean No.46?

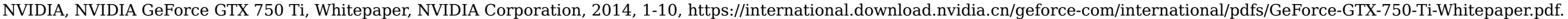

